\title{
How do abnormalities in the brain's spontaneous activity translate into symptoms in schizophrenia? From an overview of resting state activity findings to a proposed spatiotemporal psychopathology
}

Georg Northoff $\left(1,2,3,4,5,{ }^{*}\right)$, Niall W. Duncan $(3,4,5)$

1.Mental Health Centre, Zhejiang University School of Medicine, Hangzhou, Zhejiang Province, China

2. University of Ottawa Institute of Mental Health Research and University of Ottawa Brain and Mind Research Institute, Ottawa, Canada

3. Centre for Cognition and Brain Disorders, Hangzhou Normal University, Hangzhou, China

4. Brain and Consciousness Research Centre, Shuang Ho Hospital, Taipei Medical University, Taipei, Taiwan

5. Graduate Institute of Humanities in Medicine, Taipei Medical University, Taipei, Taiwan

* Corresponding author

Dr. Georg Northoff, MD, PhD, FRCP

Mental Health Centre $/ 7^{\text {th }}$ Hospital

Zhejiang University School of Medicine, Hangzhou

Tianmu Road 305,

Hangzhou, Zhejiang Province, 310013

China.

And:

Research Director

Mind, Brain Imaging and Neuroethics

Canada Research Chair

EJLB-Michael Smith Chair for Neuroscience and Mental Health

Royal Ottawa Healthcare Group

University of Ottawa Institute of Mental Health Research

1145 Carling Avenue, Room 6467

Ottawa, ON K1Z 7K4

Tel.: (613) 722-6521 Ext. 6870

Fax: (613) 798-2982

Email: georg.northoff@theroyal.ca

Website: www.georgnorthoff.com 


\begin{abstract}
Schizophrenia is a complex neuropsychiatric disorder with a variety of symptoms that include sensorimotor, affective, cognitive, and social changes. The exact neuronal mechanisms underlying these symptoms remain unclear though. Neuroimaging has focused mainly on the brain's extrinsic activity, specifically task-evoked or stimulus-induced activity, as related to the sensorimotor, affective, cognitive, and social functions. Recently, the focus has shifted to the brain's spontaneous activity, otherwise known as its resting state activity. While various spatial and temporal abnormalities have been observed in spontaneous activity in schizophrenia, their meaning and significance for the different psychopathological symptoms in schizophrenia, are yet to be defined. The first aim in this paper is to provide an overview of recent findings concerning changes in the spatial (e.g., functional connectivity) and temporal (e.g., couplings between different frequency fluctuations) properties of spontaneous activity in schizophrenia. The second aim is to link these spatiotemporal changes to the various psychopathological symptoms of schizophrenia, with a specific focus on basic symptoms, formal thought disorder, and ego-disturbances. Based on the various findings described, we postulate that the spatiotemporal changes on the neuronal level of the brain's spontaneous activity transform into corresponding spatiotemporal changes on the psychological level which, in turn, leads to the different kinds of psychopathological symptoms. We consequently suggest a spatiotemporal rather than cognitive or sensory approach to the condition, amounting to what we describe as "Spatiotemporal Psychopathology".
\end{abstract}




\section{Introduction}

Schizophrenia is a complex psychiatric disorder that includes a variety of different symptoms. People with schizophrenia can experience perceptual alterations, such as auditory hallucinations, as well as motor symptoms ranging from dyscoordination to, in extreme cases, muscular rigidity and fixed postures known as catalepsy (P. Allen et al., 2012; Daniel C Javitt \& Freedman, 2014; Northoff, 2002). In addition, there can be affective symptoms, with a blunting of emotional responses, and cognitive symptoms such as thought disorder and paranoid delusions. Finally, symptoms can include ego-disturbances, where patients describe an altered sense of self (Frith, 1992; Leube, Whitney, \& Kircher, 2008).

Various theories have been put forward to explain these disparate symptoms. In the case of sensoryperceptual symptoms, a prominently proposed mechanism is a failure in top-down cognitive processes, like the internal monitoring of speech (see (P. Allen et al., 2012; Frith, 1992)). This can be contrasted with alternative theories that posit alterations in bottom-up sensory mechanisms to explain these symptoms (D. C. Javitt, 2009; Daniel C Javitt \& Freedman, 2014). Others have preferred a more phenomenological approach that targets the patients' perceptions and experiences of their own self, body and environment, as well as of space and time (Fuchs, 2013; Parnas, Sass, \& Zahavi, 2012; Stanghellini et al., 2014; Stanghellini et al., 2016). Alternatively, explanations for symptoms such as auditory hallucinations have focused more on altered affective functions (Panksepp, 2004) or on social factors (Hoffman, 2007). However, despite impressive progress in recent years based on these theories, it remains an open question as to what specific neural mechanisms may underlie the different clusters of symptoms seen in schizophrenia.

Much of the progress in understanding schizophrenia has been based on studies employing different neuroimaging techniques. These can be applied to study the brain responses to particular stimuli or tasks, identifying how these responses differ between healthy participants and schizophrenic patients. This approach applies what has been termed an extrinsic view of the brain, as the neural activity being studied is taken to be largely determined by the external experimental input. More recently, the activity in the brain that is ongoing independent of external stimuli has become a key focus of research, providing instead an intrinsic view of the brain (Northoff, 2012, 2014; Northoff, 2014a; Raichle, 2009, 2011, 2015)(. This spontaneous activity is often studied in the so-called resting state, where participants are asked to lie still and not focus on anything in particular while the scanning goes on. It must be distinguished from this particular psychological state, however, 
and so in the following the term spontaneous activity will be reserved for the purely neuronal feature of continuous activity (see (Raichle, 2015)) while "resting state" will be used in reference to the particular psychological state present in such experiments (See (N. K. Logothetis et al., 2009; Weinberger \& Radulescu, 2015)).

The brain's spontaneous activity is structured both spatially and temporally. Spatially, spontaneous fluctuations in different brain regions are correlated with each other, forming functional networks such as the default-mode network (DMN; including regions such as the medial prefrontal cortex, the posterior cingulate cortex, and the precuneus), the central executive network (CEN; including regions including the bilateral lateral frontal cortex and the parietal cortex), and sensory networks, amongst others (Smith et al., 2009). Each of these networks is apparently more closely related to different functions, such as internally-oriented thought with the DMN, goal-oriented cognitive functions with the CEN, and sensory processing with the sensory networks. The correlated activity patterns within these different networks also interact with that in other networks to varying degrees, creating a dynamic system across the whole brain (A. C. Chen et al., 2013)). In the temporal domain, spontaneous activity patterns can be characterised by fluctuations in different frequency ranges from 0.001 to $180 \mathrm{~Hz}$. The activity in each of these different ranges can in turn be coupled in different ways, including coupling between different frequencies (cross-frequency coupling; CFC) and coupling between different sources within the same frequency. These couplings between different frequencies provide temporal structure within intrinsic activity patterns (Aru et al., 2015; Scheeringa, Mazaheri, Bojak, Norris, \& Kleinschmidt, 2011) . Importantly, studies have consistently shown major alterations in the temporal and spatial properties of activity in schizophrenia. This then raises the possibility that it is these alterations that underlie the different symptoms seen in the condition.

In more detail, we propose that spatiotemporal changes in spontaneous activity in schizophrenia result in corresponding changes in the person's perception and experience of the world. These changes may become manifest in altered experience of the self, the body, and of the temporal and spatial aspects of the environment. Such abnormalities may then translate into the various psychological symptoms seen in schizophrenia across sensory, cognitive, motor, affective, and social functions. We thus conceive of the psychopathological symptoms in schizophrenia primarily as spatiotemporal abnormalities of the brain's spontaneous activity and its spatiotemporal structure (Figure 1). This amounts to what we describe as "Spatiotemporal Psychopathology" as has already been proposed for depression (Northoff, 2015a; Northoff, 2016) (Northoff, 2015a; Northoff, 2016, 
2016a, 2016b) and bipolar disorder (Martino et al., 2016).

\section{Figure 1}

The aim of this paper is to set out the evidence for a theory linking schizophrenic symptoms to alterations in the spatial and temporal properties of the brain's spontaneous activity. While various such temporal and spatial abnormalities in intrinsic activity in schizophrenia have been observed, their exact meaning for psychopathological symptoms remains unclear (see, for instance, (Weinberger \& Radulescu, 2015)). By setting out a general theory, we aim to begin to address this shortcoming by linking these abnormal temporal and spatial features to specific symptoms. In setting out such spatiotemporal theory we will focus upon a select range of basic symptoms (Klosterkötter, Hellmich, Steinmeyer, \& Schultze-Lutter, 2001; Schultze-Lutter, 2009), abnormal time perception (Fuchs, 2013; Stanghellini et al., 2016), cognitive changes(Barch \& Sheffield, 2014; Sheffield \& Barch, 2016), ego-disturbances (Ebisch \& Aleman, 2016; Frith, 1992; Leube et al., 2008), and abnormal body perception (Northoff \& Stanghellini, 2016; Stanghellini et al., 2014) for reasons of brevity. This will leave out other core symptoms, such as auditory hallucinations and delusions (see (Alderson-Day et al., 2016)) for a recent review of resting state findings in hallucination), as being beyond the scope of the work.

The paper will discuss findings obtained using multiple neuroimaging techniques, namely fMRI, EEG, and MEG. Between them these techniques give measures of neural activity ranging from the infraslow frequency domain (fMRI; 0.01-0.1Hz), through the slow (EEG/MEG; 0.1-1Hz), and to the fast (EEG/MEG; 1-180Hz). It must be noted, however, that these different techniques measure different aspects of neural activity. BOLD fMRI provides an indirect measure of neural activation from such activation's neurovascular influence on local blood oxygen levels (Nikos K Logothetis, Pauls, Augath, Trinath, \& Oeltermann, 2001), while EEG and MEG predominantly measure voltage changes (or the magnetic changes associated with these in the case of MEG) resulting from postsynaptic ion movements from large groups of neurons with correlated activity. Recent studies have aimed to bridge the gap between these different techniques and frequency ranges. These show that infra-slow fluctuations in fMRI correspond with infra-slow fluctuations in EEG (Hiltunen et al., 2014; Monto, Palva, Voipio, \& Palva, 2008). Additionally, the infraslow fMRI signals also correlate with higher frequencies, including alpha (positive correlation), as well as beta and gamma power (negative correlation) (Scheeringa, Koopmans, van Mourik, Jensen, \& Norris, 2016; Scheeringa et al., 2011)... 
We will firstly focus upon alterations in the temporal structure of spontaneous activity in schizophrenia and how these relate to sensory, motor, and cognitive symptoms. We will then describe the changes in the spatial structure that have been observed and how these relate to thought- and ego-disorders. Each section will review the evidence accumulated to date, following which we will present some hypotheses in which we describe potential links between spatiotemporal alterations and particular schizophrenic symptoms for which there is not yet clear empirical support. We will conclude with a discussion of how our proposals fit with other theories and of future challenges for the study of the neural mechanisms underlying schizophrenia in line with the concept of spatiotemporal psychopathology. With the focus on spatiotemporal features of intrinsic activity properties, there is a large variety of other areas of schizophrenia research that will not be touched upon. For example, the extensive literature on task-evoked activity changes or structural and molecular alterations in schizophrenia will not be closely discussed. Moreover, given the complexity of the subject, the biochemical bases of the discussed activity changes (such as changes in the GABA, glutamate, and dopamine systems will not be extensively discussed. It is proposed, however, that the spatiotemporal alterations described here may represent an integrative concept that, in the future, will help to bind the findings in these other areas (See for instance (G Northoff \& E Sibille, 2014)) for linking molecular, biochemical, regional-network, and symptom levels the case of depression).

\section{The temporal structure of spontaneous activity and psychopathological symptoms}

Overall neural activity is to a large extent composed of oscillations and fluctuations occurring at different frequencies (Buzsaki, 2006). The different frequency ranges at which activity occurs have been divided into a number of broadly accepted classes (see Figure $2 \mathrm{~A}$ for these categories). The different ranges appear to have discrete sources and to be involved in somewhat different aspects of brain function. The different bands do not operate independently, however, as there are continuous interactions between them. Such interactions can be termed cross-frequency coupling, which takes a number of different forms (see (Aru et al., 2015), for a review; see also Figure 2B). In the following section we will mostly discuss how spontaneous activity occurring at these different frequencies is altered in schizophrenia, along with alterations in the interactions between the bands. We will then link these changes to particular groups of symptoms associated with the condition.

\subsection{Temporal disbalance and temporal fragmentation}

As described, neural activity is structured across a wide range of frequencies. We can assume that 
healthy functioning requires that there be a balance between the different frequencies so that no single one predominates to allow effective function. In schizophrenia, however, there is evidence that there is a disbalance in the activity levels within the different frequency bands.

Using EEG and MEG, it has been shown that there is an increase in power in the delta, theta, and alpha bands in schizophrenia ((Hanslmayr et al., 2013; Kam, Bolbecker, O'Donnell, Hetrick, \& Brenner, 2013; Kim et al., 2014; Ranlund et al., 2014)). The increase in delta power appears to occur in particular across frontal regions (Narayanan et al., 2014). In addition to these lower frequency alterations, there is evidence that gamma-band activity is increased during the resting state, particularly in cortical auditory regions, (Andreou et al., 2015; Hirano et al., 2015; Kikuchi et al., 2011; Spencer, 2011; R. White \& Siegel, 2015). This increase in resting gamma power may then cause the well established deficit in task-evoked gamma responses seen in schizophrenia (e.g., (J. M. Ford et al., 2012; L. Sun et al., 2013; P. J. Uhlhaas et al., 2006; P. J. Uhlhaas \& Singer, 2010)). Alterations in task-evoked responses have also been reported in the theta, alpha, and beta bands (e.g.,(Garakh et al., 2015; Hong et al., 2008; Kirihara, Rissling, Swerdlow, Braff, \& Light, 2012; Tan, Lana, \& Uhlhaas, 2013)). Reductions in activity strength within very low frequency bands (0.01-0.08 Hz), as measured with fMRI, have also been observed (Meda et al., 2015). These were localised to posterior cortical midline regions, with increases in power instead seen in subcortical and temporal regions. There further seems to some distinction in effects between specific ranges within these infra-slow bands, with Yu et al. (Yu, Shen, Zeng, Ma, \& Hu, 2013) reporting specific abnormalities within the slow-4 band (0.027-0.073 Hz), as opposed to slow-5 (0.01-0.27 Hz).

As well as a disbalance in activity across the frequencies, studies suggest that cross-frequency coupling is also affected in schizophrenia. Phase synchrony has been shown to be reduced in the beta band in particular (P. J. Uhlhaas et al., 2006), while reduced cross-frequency coupling between alpha and gamma bands has also been reported ((Peter J Uhlhaas \& Singer, 2012; T. P. White et al., 2010) for a review). Decreased coupling between increased beta $(15-15 \mathrm{~Hz})$ power and decreased gamma $(60-120 \mathrm{~Hz})$ power during sensory processing has been observed in schizophrenic patients (L. Sun et al., 2013; M. Sun et al., 2013). Looking at overall cross-frequency modulation, Allen (M. Allen et al., 2013) observed significantly decreased cross-frequency modulation/coupling in schizophrenia across all electrodes. However, when considering specifically fronto-temporal electrodes, schizophrenic patients showed increased cross-frequency modulation when compared to healthy subjects. Finally, a number of simultaneous EEG-fMRI studies have provided evidence for altered links between infra-slow fluctuations and both slow (delta and theta; (Razavi et al., 2013)) 
and high frequency fluctuations (gamma; (Leicht et al., 2016)).

Taken together, these findings point towards an altered global temporal structure of neural activity in schizophrenia. Firstly, there seems to be a disbalance between relatively stronger slow (delta, theta) and relatively weaker faster (beta, gamma) frequencies. This entails that the balance between them is shifted towards the longer phase durations of the former at the expense of the shorter phase durations of the latter. There is thus what can be described as a 'temporal disbalance' in the spontaneous activity's local temporal structure in schizophrenia. Secondly, such an abnormal disbalance towards low frequency fluctuations seems to go hand in hand with a decoupling between low and high frequencies, as evinced by decreased cross-frequency coupling. Accordingly, the spontaneous activity's temporal structure seems to not only be skewed towards slower frequency fluctuations, but to also show decreased relationships between different frequency bands. This amounts to what can be described as 'temporal fragmentation' in the global spontaneous activity's temporal structure.

\subsection{Temporal integration in healthy and disease}

\subsubsection{Temporal correspondence and continuity in the healthy brain}

We receive constant input from the environment in different modalities (visual, auditory, tactile, etc). At the same time, we have constantly ongoing cognitive processes and references to memory. Each of these different inputs requires integration into a whole to create the united presence we generally experience. The properties of these different systems vary, however, such as the different sensory modalities having different temporal thresholds at which discrete stimuli can be perceived (Zampini, Guest, Shore, \& Spence, 2005). This fact points towards there being a fundamental level of activity organisation that operates prior to the actions of specific sensory modalities or cognitive functions - an "amodal representational space" (van Wassenhove, 2009) - that acts to structure the activity imposed upon it. Constantly ongoing and spanning the whole brain, spontaneous activity represents a potential locus for such a fundamental process and may represent the neural manifestation of the required amodal and domain-independent representational space. As such, this activity may impact upon and give structure to all sensory and cognitive processing superimposed upon it.

This idea of such an amodal representational space can be illustrated by the example of cross-modal integration. This is the process whereby stimuli in different modalities (such as an auditory and a visual stimulus) presented at approximately the same time are bound together into consciousness. 
Where the two stimuli are presented non-simultaneously but with only a short interval between them (e.g., $<100 \mathrm{~ms}$ ) they will be mistakenly experienced as occurring at the same time. As the time period between the two stimuli becomes longer (e.g., > $150 \mathrm{~ms}$ ), the stimuli become correctly perceived as being asynchronous. The length of time between stimuli required for them to be perceived as asynchronous for individuals is known as the temporal binding window (TBW; (Dixon $\&$ Spitz, 1980)). This window can be seen as a measure of sensory integration, reflecting the temporal range within which different uni- or multi-modal stimuli can still be integrated into a single percept. The processes underlying this window (and individual differences in it) can thus be seen as forming the background upon which the modality specific processes are presented.

\subsubsection{Temporal disbalances and disrupted cross-modal integration in schizophrenia}

As described above, there appears to be a shift towards lower frequency fluctuations in schizophrenia. With such a shift we might expect that temporal binding windows in people with the condition be longer than those seen in healthy participants.

This is in fact what is seen in schizophrenia, where patients show binding window lengths in simultaneity judgement tasks of up to 100 to 150 ms longer than are seen in controls (Giersch, Lalanne, Van Assche, \& Elliott, 2013; Martin, Giersch, Huron, \& van Wassenhove, 2013). Similar findings have also been reported in tasks where the order in which stimuli are presented must be reported. In these, schizophrenic patients are unable to judge the order of presentation at stimulus interval lengths at which healthy participants are able to do so easily (Capa, Duval, Blaison, \& Giersch, 2014). Finally, in addition to these problems with temporal order and simultaneity judgements, patients with schizophrenia also show deficits in temporal prediction (Turgeon, Giersch, Delevoye-Turrell, \& Wing, 2012). This means that they are unable to detect when a series of stimuli begin to deviate from the temporal regularity that has been experienced previously.

We can speculate here that in, for example, the temporal order task (Capa et al., 2014), the two stimuli must be presented in different phase cycles of the ongoing low frequency activity for them to be perceived as distinct. In healthy participants these phase cycles occur at a frequency such that the gap between stimuli is long enough for them to be likely to fall in separate ones. If, however, the predominant frequency is shifted lower, as is the case in schizophrenia, the two stimuli will have a greater chance of landing within a single cycle and so will be perceived as a single stimulus.

Although there have not been any studies directly testing the link between altered low-frequency 
fluctuations and temporal deficits in schizophrenia, the conjunction described is highly suggestive. Importantly, the problems described are unlikely to be due to "higher-level" cognitive defects related to the making of judgements per se as the task targets are either merged or discriminated experienced percepts (Martin et al., 2013). This means that the effects must be related to more basic issues of temporal processing. Here it is possible that this issue is a disruption of the fundamental amodal and domain-independent representational space manifest in spontaneous activity, as is posited here (see Figure 3).

\section{Figure 3}

\subsection{Temporal disbalances and basic symptoms}

In the prodromal phase of schizophrenia, sufferers can experience what are collectively termed "basic symptoms", as are operationalised in the Bonn Scale for the Assessment of Basic Symptoms (Klosterkötter, Ebel, Schultze-Lutter, \& Steinmeyer, 1996; Klosterkötter et al., 2001; SchultzeLutter, 2009). These reflect early psychopathological symptoms in different domains (sensory, affective, cognitive, etc.) and generally manifest prior to the onset of an acute outbreak. In the following section we will discuss a number of these symptoms in reference to the temporal structure of spontaneous activity to outline possible links.

The Bonn scale describes several perceptual abnormalities in different modalities (visual, auditory, gustatory, olfactory, somatosensory; symptoms F1-6, O4-7) that show common, amodal underlying disturbances. In each modality, patients commonly report more intense perceptual contents, such as more intense colours or louder sounds (Martin et al., 2013). Although these experiences have not been directly linked experimentally to neural properties, some research in people at high risk of schizophrenia (who are likely to show some degree of basic symptoms) has demonstrated a reduction in evoked gamma band activity and disturbed cross-frequency coupling (Leicht et al., 2016). These findings fit in with other studies showing abnormalities in early sensory processing in schizophrenia (see (Hirano et al., 2015; Daniel C Javitt \& Freedman, 2014; Daniel C Javitt \& Sweet, 2015) for recent reviews).

Such reductions in perception-related high frequency activity may be tied to a linked to the suggested shift to low frequency activity in schizophrenia. Some indirect evidence for this comes from a recent study of emotional tone recognition (Kantrowitz et al., 2015). As expected schizophrenic patients showed impairments in auditory emotional tone recognition (behaviourally), 
along with alterations in EEG mismatch negativity that were related to dipoles in the auditory cortex and insula. Resting state fMRI in the same patients then showed reduced functional connectivity between these two regions. These results suggest that task-evoked early sensoryrelated abnormalities are related to abnormal low-frequency spontaneous activity.

In addition to perception and the sensory domain, basic symptoms also surface in the motor domain. Subtle motor symptoms are often described as neurological soft signs (Walther, 2015; Walther \& Strik, 2012); , while the most extreme motor alterations can result in catatonia (Northoff, 2002; Northoff, Kotter, et al., 2004)). The Bonn Scale describes subtle motor abnormalities as manifest in lack of coordination, motor blockages, and loss of automatic skills (symptoms O 9-11). Links between these motor abnormalities and temporal changes are suggested by the experiences of patients: (i) "I have to think of what I am going to do all the time and that takes up a lot of energy and when I am doing something I am aware of my every movement.", (ii) "When I move quickly it is a strain on me. Things go too quickly for my mind. They get blurred and its like being blind. It is as if you were seeing a picture one moment and another picture the next.", (iii) I found recently that I was thinking of myself doing things before I would do them. If I am going to sit down, for example, I have got to think of myself and almost see myself sitting down before I do it. It's the same with other things like washing, eating, and even dressing - things that I have done at one time without bothering...." ((Fuchs, 2007) p 233).

Indirect experimental support can be seen in a set of recent fMRI studies of sensorimotor function in schizophrenia. These show reductions in infra-slow frequency activity temporal variability within sensorimotor regions, as well as disrupted functional connectivity from these regions (Kaufmann et al., 2015); although note that a separate study reported an increase in variability in schizophrenia, (Yang et al., 2014). This disruption in sensorimotor functional connectivity to other regions was also seen in a separate study (Berman et al., 2015). Although primarily a spatial measure, functional connectivity has a temporal component as it involved the inter-relationship between two timeseries. Unfortunately, these studies did not look at basic symptoms, but one can suggest that these temporospatial alterations in sensorimotor regions will be involved in producing these. Future work looking more closely at the temporal properties of activity within these regions and particular symptoms is required to test this idea. In particular, such studies may want to link the temporal properties of spontaneous activity in these regions with the perception of self-initiated movement and action generation. As in the case of the binding of two stimuli into one percept (see above), one would expect a direct correspondence between time intervals on the neural level (i.e., peak 
frequency phase durations) and the perceived temporal delays in the self-initiated movements or actions.

\subsection{Decreased cross-frequency coupling and cognitive symptoms}

\subsubsection{Gamma decrease and working memory impairment}

A number of cognitive symptoms, such as working memory problems, are manifest in schizophrenia. A number of authors have suggested that this group of symptoms can be explained by a smaller number of fundamental cognitive issues. For example, Barch and colleagues argue that the inability to actively represent goal-directed information is the basic mechanism that commonly underlies the various cognitive deficits in context processing, working memory, and episodic memory in schizophrenia (see for example,(Barch \& Ceaser, 2012; Barch \& Sheffield, 2014)). Such an inability to represent goal-directed information is well established in schizophrenia and has been associated with a decrease in high-frequency, gamma band, task-induced responses in the dorsolateral prefrontal cortex (see (Lewis, Curley, Glausier, \& Volk, 2012) for an excellent overview).

These observations of reduced gamma responses can also be seen in the context of our proposed spatiotemporal approach to schizophrenia as they may reflect a change in cross-frequency coupling between low and high frequency activity. Evidence that such a mechanism is related to basic cognitive symptoms can be gained, firstly, from studies of healthy participants. In such participants, alpha-gamma cross-frequency coupling from posterior to anterior regions plays a central role in maintaining visual information in short-term memory (Pinal, Zurrón, Díaz, \& Sauseng, 2015). A decrease in this coupling with age is correlated with a decline in the ability to maintain visual information. Cross-frequency coupling has also been suggested to be important in working memory (Roux \& Uhlhaas, 2014), where, for example, low-to-high frequency coupling in the hippocampus is correlated with working memory performance (Axmacher et al., 2010).

The link between spontaneous gamma activity and working memory performance has also been tested in schizophrenic patients. In healthy participants these two measures are correlated; in patients, however, there is a reduction in gamma power and the power level no longer correlates with working memory performance. In patients, reduced working memory performance was instead associated with increased frontal alpha to gamma coupling (Popov et al., 2015). Altered coupling between infra-slow and higher frequency fluctuations is also seen in schizophrenia. Razavi (Razavi et al., 2013) report that while in healthy participants infra-slow fluctuations are correlated with 
alpha, beta and gamma activity, in patients they were instead correlated with slower frequencies (delta and theta). Finally, it can be noted that Fryer (Fryer et al., 2015) report that poorer working memory performance is correlated with the amplitude of infra-slow frequency fluctuations in regions such as the mPFC and DLPFC (components of the CEN) in both healthy controls and schizophrenia patients.

These findings suggest that the neural network underlying working memory may be abnormally dominated by slow frequencies, rather than have an appropriate balance between these and higher frequencies. The loss of coupling to faster frequencies (and their replacement by slower frequencies) may indicate that shortly presented stimuli at faster frequency ranges can no longer be linked to the longer cycle durations of the slow and especially infraslow frequencies; this in turn may make impossible their maintenance in working memory as it may be specifically related to the longer cycle durations of the infraslow frequencies. One may hypothesize that the degree of decoupling between faster and especially infraslow frequencies may be directly proportional to the duration in which a stimulus or event can be maintained in working memory: the higher the degree to which the faster frequencies are decoupled from the slow and infraslow ones, the less can a stimulus or event be linked and integrated to the long cycle durations of the infraslow frequencies, and the shorter the duration for which that very same stimulus or event can be maintained in working memory.

\subsubsection{Changes in slow frequencies and attention deficits}

As well as working memory performance, a relationship between altered temporal properties of activity and attentional performance has also been reported in schizophrenia. In line with other work, (L. Chen et al., 2015) reported that delta and theta power was elevated in patients, localised to the right frontal and right temporoparietal cortices (elements of the attention network). This increase was correlated with worse attentional performance in these patients (as well as in the controls, arguing for a continuum-like effect). In addition to increased theta power, a reduction in the coupling between this band and gamma activity has been related to impaired attention in schizophrenia (Popov et al., 2015), where theta phase in the anterior cingulate was less coupled with gamma power in lateral regions.

Finally, poor sustained attention was found to be correlated with reduced variability in infra-slow activity in regions including the left frontal cortex, bilateral temporo-parietal junction, anterior and 
posterior cingulate cortex, and right dorsolateral prefrontal cortex (Fryer et al., 2015). These regions for parts of the dorsal and ventral attention networks (Corbetta, Patel, \& Shulman, 2008; Corbetta \& Shulman, 2011). This decrease in variability at the neural level may map onto cognitive-attentional functions where it could manifest in a slowing down of thought, as is reported in schizophrenia. This is speculation at present but could be tested by using self reports of thought changes to develop a behavioural frequency spectrum that could then be mapped onto alterations in the frequency ranges of spontaneous activity in the brain.

\subsection{Global temporal fragmentation of spontaneous activity and generalized cognitive symptoms} In the previous sections we have described links between particular schizophrenic symptoms and alterations in brain activity that are associated with them. We have described how alterations in the brain's intrinsic activity's local temporal structure may underlie these disparate symptoms through interruption of the normal relationships between temporal features. In the following paragraphs we will extend this framework to the global temporal structure of the brain's spontaneous activity (see for instance Yang (Yang et al., 2014; Yang et al., 2016) observing global changes in neural activity) and link it to generalized cognitive changes (e.g., cognitive dysmetria and disrupted time perception) in schizophrenia as a set of hypotheses that remain to be tested.

\subsubsection{Global temporal discoordination in spontaneous activity and generalized cognitive changes}

Early psychiatrists such as Kraepelin and Bleuler postulated a basic deficit in schizophrenia that underlies its various symptoms. Kraepelin (Kraepelin \& Diefendorf, 1915), for instance, suggested that the primary deficit in schizophrenia is a lack of coordination and organisation; that it is like an orchestra without a conductor. Given the findings reported here one may view the brain's spontaneous activity as such a conductor, providing temporal structure whose absence leads to schizophrenia. Bleuler (E. Bleuler, 1911) assumed that schizophrenia and its various symptoms stem from a deficit in association between the different contents in our perception and cognition: there is a "loosening of association" in the basic cognitive processes which strongly impacts coordination and organisation of the contents in subsequent cognitive, affective, sensory, and motor functions.

The lack of organisation and coordination resurfaces in the more recent theory of N. Andreasen, who characterizes schizophrenia by what she describes as "cognitive dysmetria"(Andreasen, 1999; Andreasen et al., 1999). In this it is proposed that the lack of temporal coordination in thought and cognition can be traced to a neuronal deficit in the cortical-cerebellar-thalamic-cortical circuit 
(CCTCC; see (Sheffield \& Barch, 2016), for a review of alterations in functional connectivity in this circuit in schizophrenia). Encompassing more or less the whole brain, the CCTCC temporally organizes and structures cognition, accounting for what Andreasen describes as synchrony. Deficits in the CCTCC, as in schizophrenia, may then result in disruption to the temporal organisation and structuring of cognition, as it is manifest in many symptoms in sensory, motor, cognitive and even affective domains.

The assumption of deficits in especially the cerebellum and its relationship to the cortex and thalamus is indeed supported by recent imaging studies. Shinn(Shinn, Baker, Lewandowski, Öngür, \& Cohen, 2015), using resting state fMRI, focused on the functional connectivity of the cerebellum to various network in the cortex. They observed reduced functional connectivity of the cerebellum to regions in various networks (executive network, default-mode network, ventral attention network, salience network) in schizophrenia patients. This was accompanied by increased functional connectivity from the cerebellum to other regions in the DMN and sensorimotor network (See also (Anticevic et al., 2014; Wang \& Krystal, 2014) and (A. C. Chen et al., 2013) for more or less analogous findings).

Are these changes in the cerebellum and cortex related to cognitive deficits? Matsuo (Matsuo et al., 2013) conducted an imaging study during a verbal working memory task. They observed decreased activation in cerebellum (vermis), default-mode network, pons, thalamus and lateral prefrontal cortex in schizophrenia. Wagner (Wagner et al., 2015) reported reduced activity and functional connectivity in cerebellum, thalamus and anterior cingulate in schizophrenic patients during the Stroop interference task where patients showed severe attentional deficits. Finally, Bernard and Mittal(Bernard \& Mittal, 2014b) (see also (Bernard \& Mittal, 2014a)) conducted a meta-analysis of imaging studies in various domains. They observed that the cerebellum shows deficits in schizophrenia, particularly in the domains of working memory and emotions. They suggest therefore a central role of the cerebellum in schizophrenia in that it provides abnormal internal models that are then used as templates in motor, cognitive, affective domains. Taken together (See (Bernard \& Mittal, 2014a, 2014b) for reviews), these findings indicate that cerebellar-thalamocortical changes may be central in cognitive symptoms in particular, close to the idea of cognitive dysmetria.

How can we reconcile the assumed deficits in cerebellar-thalamo-cortical circuits with the observation of temporal fragmentation in the spontaneous activity's temporal structure? Operating 
across the whole brain in an amodal and domain-independent way, temporal fragmentation may affect the whole brain, thus operating across the various networks and regions discussed, including the cerebellum. One may consequently assume that the observed changes in resting state cerebellarthalamo-cortical networks and regions may result from an altered underlying global temporal structure, with global temporal fragmentation in the "amodal and domain-independent representational space" (see above) of the brain's spontaneous activity. For instance, the lack of cross-frequency coupling with subsequent global temporal fragmentation in the spontaneous activity's temporal structure may then be manifest spatially in decreased cross-regional coupling, and ultimately in what Andreasen describes as cognitive dysmetria.

The assumption of a global change in neural activity and its association with cognitive deficits is supported by recent studies in both EEG and fMRI. Several EEG observed increases in global noise power (as measured by decreased signal-to-noise ratio) in pre- and post-stimulus activity (see (Molina et al., 2016; Suazo et al., 2016; Winterer et al., 2000)). Such increases (as measured in slow and fast frequencies in EEG) is compatible with the increase in global power and variability and functional connectivity as observed in the infraslow frequency range in fMRI by Yang (Yang et al., 2014; Yang et al., 2016). The increased noise affects the degree of stimulus-induced activity (with higher noise leading to lower amplitudes; Molina (Suazo et al., 2016) and impairs cognition (with higher noise leading to lower cognitive performance; (Diez, Suazo, Casado, Martin-Loeches, \& Molina, 2013; Suazo et al., 2016).

\subsubsection{Global temporal fragmentation and perceiving the world as "snapshots"}

What happens if the spontaneous activity's global temporal structure is altered as in schizophrenia with decreased cross-frequency coupling? In that case the extremely short cycle durations of faster frequencies can potentially no longer be linked and integrated with the longer ones in the infraslow frequency ranges. This means that different stimuli of different durations can no longer be integrated and linked. They are consequently perceived as temporally segregated without any perceptual continuity between them - what is described as the "stream of consciousness" becomes disrupted and dissolved into different "snapshots", entailing temporal fragmentation in both sensory and cognitive domains (See Figure 4 for a general overview).

\section{Figure 4}

Such temporal fragmentation, with a disruption of cognition into "snapshots", is, for instance, 
manifest in the symptom of thought blocks, where patients suddenly stop talking and thinking. A recent study by Angelopoulos (Angelopoulos, Koutsoukos, Maillis, Papadimitriou, \& Stefanis, 2014) used EEG to investigate brain activity during thought blocks in schizophrenic patients with auditory verbal hallucinations. Thought blocks were characterized by patients as "a rock in my head", "blank page in my mind", "spontaneous block of my thought", and "loss of mind" which usually occurred automatically during the hallucinatory state. In EEG it was observed during these thought blocks that there was significantly decreased phase synchrony for about 0.5 to $2 \mathrm{~s}$ in theta and alpha range $(6-12 \mathrm{~Hz})$ with abnormal coupling between left temporal and frontal electrodes. As well as this, whole brain synchrony entropy (a measure of the complexity of phase synchrony between regions over time) was reduced during thought blocks, indicating a stereotypical reduction in synchrony across the brain, normalising upon recovery. The authors suggest that the low phase synchrony and synchrony entropy mean that the brain is unable to process information, resulting in the thought block symptoms. This may echo what we discuss here as temporal fragmentation in the spontaneous activity.

How about the manifestation of temporal fragmentation in the sensory domain and especially the perception of time itself? There have been many qualitative reports of altered subjective time experience in schizophrenia in what is described as 'Phenomenological Psychiatry' (see (Fuchs, 2007, 2013; Parnas et al., 2012) for recent overviews). However, quantitative investigation is still lacking. One recent study investigated the subjective experience of time as described by schizophrenic patients $(\mathrm{n}=301)$ and analysed these reports in a semi-quantitative way(Stanghellini et al., 2016). They reported that the main disturbance in schizophrenia (as distinguished from depression) consisted in what they described as fragmentation or "disarticulation of time experience": "The external world appears as a series of snapshots" or "world like a series of photographs" (Stanghellini et al., 2016). The lack of temporal continuity between contents in perception is also well reflected in the following quote by a schizophrenic patient by (Fuchs, 2007): "When I move quickly, it is a strain on me. Things go too quickly for my mind. They get blurred and it is like being blind. It's as if you were seeing a picture one moment and another picture the next." (Fuchs, 2007); see also, (Fuchs, 2009, 2013).

How is such abnormal perception of contents in time related to the spontaneous activity and its temporal structure? One would hypothesize that the degree of temporal fragmentation in perception of contents in terms of "snapshots", "series", "pictures" or "now" is directly proportional to the degree of loss of cross-frequency coupling especially between fast and infraslow frequency 
fluctuations: the lower the degree of infraslow-fast cross-frequency coupling, the higher the degree to which subjects perceive contents in terms of "snapshots", "series", "pictures" or "now". The temporal fragmentation on the neural level of spontaneous activity may thus map onto the perceptual level and its temporal fragmentation. Such an effect has been observed in a recent study where series of auditory stimuli were presented and the entrainment of delta activity to them measured (Lakatos, Schroeder, Leitman, \& Javitt, 2013). In healthy participants, the phase of the delta activity was shifted to correspond to the stimulus onset. This effect was not seen in schizophrenic patients, however, despite them showing task evoked activity changes to the individual stimuli. This means that the patients were able to respond to single presentations but were unable to attune their neural activity to the stimulus properties over time (i.e., the stimulus sequence) (see also (Winterer et al., 2000) for analogous findings of reduced phase-locking to external stimuli in the delta and theta range in schizophrenia). This may represent a breakdown in the processing of the continuity of stimuli from the world, similar to the experience of "snapshots" described.

\section{The spatial structure of spontaneous activity and psychopathological symptoms}

In the preceding sections we have focussed upon alterations to the temporal structure of neural activity in schizophrenia and how these relate to symptoms. We will now shift our attention to the spatial structuring of activity and how this is affected. Many of the findings discussed involve functional connectivity between different brain regions and the manner in which regions interact to form functional networks. As noted above, such functional connectivity is established through correlations between the activity timeseries within the particular regions being studied. We will begin by looking at alterations in spatial structure across the brain as a whole before then looking at changes within particular brain regions or functional networks. These will then be related to particular symptoms before we present a set of hypotheses about relationships between the spatial structure of intrinsic activity and symptoms seen in schizophrenia in the final subsection.

\subsection{Alterations in the spatial structure of spontaneous activity in schizophrenia}

\subsubsection{Global alterations in activity structure}

Global network structure across the brain, as measured with fMRI, appears to be altered in schizophrenia, with a reduction in small-worldness, number of hubs, and modularity (Karbasforoushan \& Woodward, 2012). This finding is supported by a recent study of schizophrenic patients that found a greater degree of randomisation in network metrics, plus fewer hubs and larger connection distances between hubs (Lo et al., 2015). This study also included 
relatives of the patients, in whom some of the same abnormalities were found, although to a lesser degree. These results suggest that communication across the brain as a network is less efficient (Bullmore \& Sporns, 2009). This is indeed what was found in a third study, in which network global efficiency was tested and was found to be reduced in schizophrenic patients ( $\mathrm{Su}, \mathrm{Hsu}, \mathrm{Lin}, \&$ Lin, 2015). Local efficiency did not differ between the groups. Importantly, reductions in global efficiency correlated with the severity of overall psychopathological symptoms, negative symptoms, and depression/anxiety symptoms: the lower the spontaneous activity's global path efficiency, the more severe the overall symptoms and the worse the negative and depression/anxiety symptoms.

Taken together, these findings clearly show changes in the spontaneous activity's global spatial structure in schizophrenia. The suggestion of an altered global neural organisation with disconnectivity is reminiscent of Bleuler and his earlier characterization of schizophrenia as a "loosening of associations" (E. Bleuler, 1911), with abnormal interplay between the different mental faculties. This is compatible with an assumption that schizophrenia is in part a disconnection syndrome across the whole brain (Stephan, Friston, \& Frith, 2009) or as a global connectivity disorder of the spontaneous activity's global spatial (and temporal) structure.

\subsubsection{Local alterations in activity structure}

In addition to these global abnormalities, schizophrenia patients also show more local alterations to spontaneous activity patterns within specific neural networks, along with alterations in the way different networks interact with each other. Firstly, various investigations have shown alterations to functional connectivity within the DMN. Although these have not been entirely consistent, with some showing increased connectivity (Kindler et al., 2013; Rotarska-Jagiela et al., 2010; Susan Whitfield-Gabrieli et al., 2009)) and others decreased ((Bluhm et al., 2007; Garrity et al., 2007), the most common finding is for greater connectivity and activity levels (see (S. Whitfield-Gabrieli \& Ford, 2012) and (Anticevic et al., 2012)for reviews).

In contrast to the DMN, the CEN generally shows reduced functional connectivity amongst its constitutive regions (like the lateral prefrontal and parietal cortex) in schizophrenia (e.g., (Tu, Lee, Chen, Li, \& Su, 2013; Woodward, Karbasforoushan, \& Heckers, 2012);), which is expected given the major executive deficits observed on these patients. More interestingly, the relationship between these two networks appears to be altered in schizophrenia. Whereas in healthy subjects the activity in these two networks is usually negatively correlated, in patients it is instead positively correlated, with an increased connection strength and a mixing of signals between the two ((Littow et al., 2015; 
H. Liu et al., 2012; Manoliu et al., 2014; Tu et al., 2013; Wotruba et al., 2013) . These changes in connectivity between the two networks are correlated with symptom severity (Manoliu et al., 2014; Wotruba et al., 2013). Notably, such a switch in network relation from negative to positive is also seen in heathy participants given the psychedelic drug psilocybin, which can be used to mimic psychosis (Carhart-Harris et al., 2013).

As well as changes in the DMN and CEN, alterations in other networks are also observed in schizophrenia. As discussed previously, both variability and functional connectivity appear to be altered within the sensorimotor network (Kaufmann et al., 2015; Li et al., 2015) . Again, these findings are not homogeneous, however, as other studies have reported increased connectivity between the thalamus and sensory regions in the cortex (Anticevic et al., 2014; Damaraju et al., 2014).

More consistent are findings of alterations to the spatial structure of activity within the salience network (SN). A study by Palaniyappan (Palaniyappan, Simmonite, White, Liddle, \& Liddle, 2013) investigated resting state functional connectivity with Granger causality that allows measuring causal and excitatory/inhibitory relationship between regions/networks. In healthy participants they observed excitatory functional connectivity from the right anterior insula (rAI) and the dorsal anterior cingulate cortex (dACC) to the bilateral DLPFC, while the latter showed inhibitory functional connectivity to the former. Schizophrenic subjects, in contrast, showed significant decreases in both rAI/dACC-DLPFC excitatory connectivity and DLPFC-rAI/dACC inhibitory connectivity, entailing a decoupling or dissociation between the SN and CEN. In addition, excitatory connections from the bilateral visual cortex to the hippocampus and insula showed significant decreases in schizophrenic patients. Finally, decreases in both rAI/dACC-DLPFC reciprocal connectivity and visual cortex-AI connectivity correlated with symptom severity.

These findings suggest an altered balance between the SN and CEN, with both apparently being decoupled from each other in schizophrenia. This has also been shown in other studies, where the change in the balance between the two networks is correlated with hallucination symptom severity (Manoliu et al., 2014). This study also found a reduced connectivity between the SN and DMN. Importantly, the functional connectivity of rAI correlated with increased functional connectivity between the DMN and CEN: the lower the functional connectivity of the right anterior insula, the more increased the functional connectivity between DMN and CEN. These results suggest that hypoactivity in the anterior insula is related to an abnormally strong relationship between DMN and 
CEN (see (Moran et al., 2013) for further confirmation).

As well as the DMN and CEN, connectivity between the SN and the striatum is also reduced in schizophrenia (Orliac et al., 2013). This reduced connectivity correlated with the severity of delusions (and depression). Since the striatum (as part of the salience network) is central in reward and salience processing, these results lend support to the "aberrant salience hypothesis" in schizophrenia (see for instance (Pankow, Knobel, Voss, \& Heinz, 2012)). This hypothesis postulates that schizophrenic patients attribute aberrant salience to irrelevant stimuli rather than relevant stimuli, which is assumed to be central in generating delusions. The observation of abnormal striatum spontaneous activity is also in line with a recent meta-analysis of reward activation studies that showed decreased activation in ventral striatum during specifically reward anticipation or prediction of specific stimuli in schizophrenic patients (see (Radua et al., 2015) and (Pankow et al., 2012).

Taken together, these findings show alterations in functional connectivity within and between networks in schizophrenia. Of these, alterations in DMN, salience network and CEN connectivity are most notable. Moreover, the results show altered cross-network functional connectivity, with abnormal balances between the DMN and CEN, as well as between the salience network these other two networks. Notably, the DMN-CEN balance is no longer one of anticorrelation, but is instead a positive correlation.

\subsection{Abnormalities in the spatial structure and cognitive symptoms}

\subsubsection{The abnormal balance between the DMN and CEN and formal thought disorder}

As described, there appears to be an alteration in the balance between activity in the DMN and CEN in schizophrenia. In healthy participants, the activity in these two networks is anticorrelated, meaning that high activity in the one is correlated with lower activity in the other. Particularly in anterior regions of the networks, this negative relationship is associated with a balance between internal and external mental contents (i.e., self- and environment-related contents, respectively; (A. C. Chen et al., 2013; Vanhaudenhuyse et al., 2011) in what has been described as reciprocal modulation (Goel \& Dolan, 2003a, 2003b; Northoff, Heinzel, et al., 2004).

More specifically, higher levels of activity within anterior medial regions of the DMN (i.e., the mPFC) are linked to a focus upon internal mental contents, self-directed thoughts, and to sensations of parts of their own body (like the limbs or the face) and to bodily processes (such as the heartbeat; 
(Vanhaudenhuyse et al., 2011)). In contrast, activity in the lateral regions within the CEN (e.g., the DLPFC) is associated with mental content directed towards the external environment (Georg Northoff \& Etienne Sibille, 2014). As such, the balance between the activity in these two networks transforms into a reciprocal balance between internal and external mental contents on the psychological level. In schizophrenia, however, studies show a clear shift in the balance between the two networks such that this negative correlation becomes instead positive ((Littow et al., 2015; H. Liu et al., 2012; Manoliu et al., 2014; Tu et al., 2013; Wotruba et al., 2013). This change has in addition been found to be correlated with auditory hallucination severity (Manoliu et al., 2014). This implies that internal and external mental contents are no longer reciprocally modulated either. Instead of external mental content decreasing when internal mental contents increase (and vice versa), both contents become directly and positively linked with each other.

The implication of this is that internal and external contents are no longer appropriately segregated from each other, meaning that the distinction between thought contents related to the internal self and those associated with the external environment becomes blurred. This confusion between internal and external contents is analogous to the formal thought disorder described by Bleuler (Eugen Bleuler \& Brill, 1924) as a core symptom of schizophrenia. Such disorders are manifest in symptoms such as increased indecisiveness with regard to insignificant choices between equal alternatives, thought interference, thought blockages, disturbances of abstract thinking, and a decreased ability to discriminate between ideas, perception, fantasy and true memories. We can then see how a mixing between internal and external mental contents could lead to these symptoms. For example, such a mixing could lead to indecisiveness as decision making becomes difficult when it becomes harder to differentiate between internal and external cues (see (Nakao, Bai, Nashiwa, \& Northoff, 2013; Nakao, Ohira, \& Northoff, 2012), for a discussion of internally and externally guided decision making). Similarly, thought interference may arise when the barriers between different contents are lost, leading to thought becoming an amalgamation of the two. Such a link between a shift to a positive between-network correlation and thought disorder has indeed reported (Wotruba et al., 2013), although further research in this direction is required.

\section{Figure 5}

\subsubsection{Cortical midline connectivity and ego-disturbances}

One of the most prominent symptoms in schizophrenia is ego-disturbances. Early psychiatrists, such as Kraepelin, Berze, Schneider, and Scharfetter, considered such alterations in the sense of self 
and identity to be a core characteristic of the condition (see (Leube et al., 2008) for an overview). Such symptoms have also been subsumed under the concept of passivity phenomena (Frith, 1992; Leube et al., 2008). In healthy participants there is a close link between self-related processing and anterior cortical midline structures (P. Qin et al., 2012). In schizophrenia, however, there is a change in the functioning of these regions during self-related processing.

Firstly, the spatial properties of activity within these regions appears to be altered, with greater connectivity within cortical midline regions of the DMN during self-related processing ((Ćurčić-Blake, van der Meer, Pijnenborg, David, \& Aleman, 2015; Holt et al., 2011; van Buuren, Vink, \& Kahn, 2012). At the same time, task evoked activity within these regions has generally been found to be increased during tasks that involve some form of self-referential thought ((J. Liu, Corbera, \& Wexler, 2014; Menon et al., 2011; Shad et al., 2012).

Although these studies haven't directly related these fMRI effects to ego-disturbance symptoms, Taylor and colleagues (Taylor, Welsh, Chen, Velander, \& Liberzon, 2007) compared schizophrenic patients with and without reality distortion in an emotional picture viewing task and again found increased activity in anterior midline regions in those with distortions. Similarly, there is evidence that altered activity in cortical midline regions (amongst others) is related to deficits in insight in schizophrenia, symptoms which have a strong self-referential component (Ćurčić-Blake et al., 2015; van der Meer, Costafreda, Aleman, \& David, 2010; van der Meer et al., 2013).

Taken together, these studies demonstrate clear task-evoked alterations in midline regions (core components of the DMN) during self-related processing in schizophrenia. Most studies suggest increased activity during specifically self-related processing; however, results are not homogeneous on this front. Unfortunately, none of these studies report results from the resting state in the same subjects to link this to the stimulus-induced activity during the self-reference task. One would predict that, as in healthy subjects, abnormalities in spontaneous activity the midline regions may predict the task-evoked changes, and possibly also symptoms such as reality distortion and other ego-disturbances.

Figure 6

3.3 Hypotheses: Altered spatial properties of spontaneous activity and symptoms 3.3.1 Abnormal temporal and spatial structure in the DMN and ego disturbances 
Ego disturbances in schizophrenia can be described as a breakdown in the distinction between self and the environment, (Ebisch \& Aleman, 2016; K. Schneider, 1974). These can include things such as thought insertion and feelings of being controlled by external agents. In such symptoms, stimuli which should be experienced as originating from or being related to the self are instead experienced as coming from outside sources (Ebisch \& Aleman, 2016). The loss or detachment from the own self is well reflected in the following quote from the first-person perspective: "I feel that my real self has left me, seeping through the fog toward a separate reality, which engulfs and dissolves this self. This has nothing to do with the suspicious thoughts or voices; it is purely a distorted state of being. The clinical symptoms come and go, but this nothingness of the self is permanently there. Not a single drug or therapy has ever helped with such nothingness. By nothingness, I mean a sense of emptiness, a painful void of existence that only I can feel. My thoughts, my emotions, and my actions, none of them belong to me anymore." (Kean, 2009), p.1034).

Could such symptoms be explained by alterations in spontaneous activity? Evidence that this could be the case comes from investigations in healthy participants of self-related processing. These have demonstrated an overlap between those regions that display a high degree of ongoing activity and those regions implicated in the discrimination between self and other and in determinations of selfrelatedness (P. Qin \& Northoff, 2011). In particular, regions within the DMN have been highlighted as being key to these processes (D'Argembeau et al., 2005; Lipsman et al., 2014; P. Qin \& Northoff, 2011; F. Schneider et al., 2008; Susan Whitfield-Gabrieli et al., 2011). This has been coined "rest-self overlap" (Bai et al. 2015, (Northoff, 2015).

More recent studies have built on this overlap to demonstrate that spontaneous activity fluctuations with DMN regions are related to different aspects of self-related processing. The first of these showed that the level of EEG alpha power prior to a stimulus being presented predicted whether a person would ascribe a high or low degree of self-relatedness to an emotional stimulus.

Interestingly, alpha power was correlated with glutamate concentrations within the pACC, a factor that is known to be altered in schizophrenia (Gallinat, McMahon, Kühn, Schubert, \& Schaefer, 2015; Mouchlianitis et al., 2015; Smesny et al., 2015)). A second study then showed that levels of spontaneous activity within the right temporoparietal junction (rTPJ) prior to stimulus onset, as measured with fMRI, determined whether an ambiguous sound would be experienced as the person's own name or not (Pengmin Qin et al., 2016). Where there was a higher level of spontaneous activity within the rTPJ immediately prior to the sound being presented, the sound would be ascribed self-relatedness. Finally, temporal structure of ongoing activity within the mPFC 
(as indexed by the power-law exponent) was shown in a third study to be correlated with scores for private self-consciousness in healthy individuals (Zirui Huang, Obara, Davis IV, Pokorny, \& Northoff, 2016).

Taken together, these studies suggest that there is neural overlap between spontaneous activity and self-processing, where self-relatedness may be encoded in the spatiotemporal properties of this activity. We can then see how alterations in spontaneous activity's spatial (and temporal) structure in schizophrenia could translate to ego disturbance symptoms, where ascriptions of self-relatedness for stimuli begin to break down. Where a stimulus (such as a thought or the perception of an action) should be automatically experienced as being self-related, the altered spontaneous activity properties lead it to be experienced as external to the individual. We would further hypothesise that it is not simply the properties of spontaneous activity within the highlighted DMN regions that lead to these symptoms, it is also how these regions are coupled to the rest of the brain. As described previously, there is evidence that there is an abnormal relationship between the DMN and CEN in schizophrenia. Stemming from this, we postulate that this altered communication between networks may contribute to ego disturbances as it may lead to an inability to link self-relatedness (as processed within particular DMN regions) with stimulus-related processing in other networks (see Figure 5B). The individual will therefore be unable to correctly distinguish between stimuli that should be labelled as originating from them and stimuli which should be labelled as coming from the environment.

Though tentative and preliminary, our account amounts to a spatiotemporal theory of egodisturbances that is based on spontaneous activity and its spatiotemporal structure. Such a spatiotemporal approach (see also (Robinson, Wagner, \& Northoff, 2015) must be distinguished from other theories of ego-disturbances in schizophrenia that are either bottom-up and sensorybased or, alternatively, top-down as related to cognitive functions like source monitoring (see (Mishara et al., 2015; Nelson, Whitford, Lavoie, \& Sass, 2014a, 2014b). Rather than being based on task-evoked activity as related to either sensory (as in bottom-up models) or cognitive (as in topdown models) functions, the spatiotemporal approach is more basic and fundamental than both since it is based on the spontaneous activity and its spatiotemporal structure. This makes it futile to characterize it as either bottom-up or top-down; instead one may want to speak of a basic or fundamental model of ego-disturbances analogous to the recently described "basic model of selfspecificity" in the healthy brain (Northoff, 2015) .However, future investigation is needed in order to directly compare the spatiotemporal approach with bottom-up and top-down theories to see to 
what degree they are compatible with, or, better, complementary to, each other.

\subsubsection{An abnormal relationship between functional networks and abnormal body perception}

Patients with schizophrenia can experience altered perception of their own bodies. Based on interviews with a large number of patients $(n=301)$, (Stanghellini et al., 2014) describe how many with schizophrenia experience their body as mechanised or objectified. This means that they no longer experience their body from a living, subjective perspective, but instead experience it objectively as a thing that is devoid of life ("She felt programmed like a robot"; ibid, p 1707). Importantly, these experiences were only reported in schizophrenia and not in a comparison group of depression patients $(n=56)$. In addition to these experiences of bodily objectification, patients with schizophrenia also report issues with experiencing bodily boundaries. This has been described as a "dynamization of bodily boundaries and construction" (Stanghellini et al., 2014), p 1706) and can involve experiences such as penetration by strange forces ("areas of body where forces enter"; ibid, p 1706), unusual spatial relationships ("mouth was where hair should be"; ibid, p 1706), and, in extreme cases, an externalisation of body parts ("vagina half outside"; ibid, p 1706).

A key brain region for processing stimuli originating within the body is the anterior insula (Craig, 2009b). This region forms part of the salience network (Seeley et al., 2007), a network that has altered connectivity to the DMN and CEN in schizophrenia ((Alonso-Solís et al., 2015; Iwabuchi, Liddle, \& Palaniyappan, 2015; Nekovarova, Fajnerova, Horacek, \& Spaniel, 2015)). This suggests that the body perception abnormalities experienced in schizophrenia may be due to altered spontaneous activity properties in these networks. We propose that reduced functional connectivity between the anterior insula and the anterior DMN results in a deficit in self-ascription to bodily stimuli being processed in the insula (see (Northoff \& Stanghellini, 2016)for more details). This may lead to a higher likelihood that the bodily stimuli will be experienced as coming from objects in the environment (rather than the body). As items in the environment, these objects are likely to be interpreted as non-living, leading to the experiences of mechanisation (which in turn may reduce the assignment of agency and ownership to the own body on a cognitive level; (Northoff \& Stanghellini, 2016; Robinson et al., 2015) (resulting in what phenomenologists' describe as "objective body" as distinguished from the "lived body"; (Northoff \& Stanghellini, 2016). In addition, increased connectivity between the salience network and the CEN may lead to altered perceptions of spatial relationships in respect to the body and the environment. This may lead to the jumbling of bodily relations seen in schizophrenia as the boundaries between the body and the environment become more fluid and dynamic. These hypotheses do, of course, remain to be 
investigated in future studies.

\section{Discussion}

\subsection{Spatiotemporal psychopathology in context}

How does the spatiotemporal approach put forward here stand in relation to other theories of schizophrenia? Since it is based on spontaneous activity itself, and as such is not necessarily linked to any one region or process, our approach does not contradict sensory-based bottom-up not cognitive-based top-down explanations for symptoms. Instead, it could be argued that the changes in spontaneous activity properties described provide the basis for the observed changes in both topdown and bottom-up processing. The spatiotemporal approach may thus provide a wider and more fundamental context that allows us to reconcile the apparently contradictory bottom-up and topdown approaches.

Our spatiotemporal approach also provides potential mechanistic background to neurodevelopmental approaches to schizophrenia (see, for example, (Fatemi \& Folsom, 2009; O. D. Howes \& Murray, 2014; Najas-García, Rufián, \& Rojo, 2014). Spontaneous activity properties are succeptible to both developmental and socio-contextual influences. For example, a recent study showed that negative early life experiences are correlated with both glutamate concentrations and fMRI activity entropy (i.e., how ordered the activity is over time) in the mPFC (N. W. Duncan et al., 2015). This and other studies show that spontaneous activity and its spatiotemporal structure are highly experience-dependent (see also(Sadaghiani \& Kleinschmidt, 2013), and are therefore susceptible to being shaped by environmental and developmental processes. Accordingly, suggestions of neurodevelopmental and neurosocial mechanisms in schizophrenia fit well with the here suggested spatiotemporal approach.

Other researchers have put forward phenomenological approaches to understanding schizophrenia ((Parnas et al., 2012; Stanghellini et al., 2014; Stanghellini et al., 2016), These focus upon the subjective experience of the patient from a first-person perspective. We postulate that this subjective experience is directly related to the spatiotemporal structure of spontaneous activity and the manner in which this is transformed onto the psychological level. Support for such a relationship between spontaneous activity can be found in studies in healthy participants where spontaneous activity levels determine how particular stimuli will be perceived (Sadaghiani, Hesselmann, Friston, \& Kleinschmidt, 2010). 
We would propose that the temporal and spatial changes seen in intrinsic activity in schizophrenia shape the processing of stimulus-induced activity, which in turn affects the cognitive, affective, and social processing. Such effects become manifest in particular symptoms ((Northoff \& Stanghellini, 2016). There is thus a potential common root - the spatiotemporal structure of intrinsic activity that binds both the subjective experience of the individual and the objectively observed symptomatology of the disease. In healthy participants, this common root linking intrinsic activity with experience and behaviour may be seen in findings linking self-related information to intrinsic activity, where an individual's self-related processing is seen to overlap with resting state function (see for example, (Bai et al., 2015; Z Huang, Ferri, Longtin, Dumont, \& Northoff, 2016; Nakao et al., 2013; Nakao et al., 2012; Pengmin Qin et al., 2016) ). These results, in conjunction with those previously mentioned linking intrinsic fluctuations to perceptual experience, provide preliminary evidence that the spatiotemporal structure of intrinsic activity may translate to the phenomenological-experiential level, resulting in so-called "neurophenomenal hypotheses" (Northoff, 2014b, 2014c; Northoff \& Stanghellini, 2016). Methodologically, this means that subjective reports of experiences of self, body, and the world could be considered as useful data that could lend support to the suggested spatiotemporal hypothesis about specific symptoms. Future investigations may want to focus on specifically the perception of time and space with regard to the various symptoms in order to link those spatiotemporal features to the corresponding spatial and temporal features in the spontaneous activity.

In addition to being a potential bridge between brain, experience, and associated phenomenological pathology, our proposed spatiotemporal psychopathology may also help bridge the gap between the brain, on the one hand, and cognitive (Frith, 1992) and affective (Panksepp, 2004) models of psychopathology on the other. It does this by proposing a primary functional alteration in disease that influences the temporal and spatial structuring of specific cognitive or affective contents (rather than a particular deficit in any one cognitive, affective, or sensorimotor system that generates such contents). Cognitive and affective psychopathologies are thus not seen as separate, independent pathological processes but are instead integrated under a single fundamental disease process.

Finally, one can ask how the proposed approach fits with the recently introduced RDoC classification (Cuthbert \& Insel, 2013). Like RDoC, the spatiotemporal approach views disease symptoms, and the mechanisms underlying these symptoms, as extremes on a continuum that includes the healthy state. For instance, the relationship between activity in the DMN and CEN with regard to the distinction between internal and external mental contents can range on a continuum 
from an extremely negative (as in depression; (Northoff, 2016a, 2016b) to abnormally positive (as in schizophrenia; see above) correlation (see 3.1.2. for details). In-between these extremes, one finds healthy subjects, who seem to show a medium or average anti-correlation value in DMN-CEN relationship.

Together, this amounts to an inverted U-shape curve (see also (He \& Zempel, 2013)) that describes the continuum of different DMN-CEN correlation values across healthy and psychiatric subjects in a dimensional way. An analogous inverted U-shape can be drawn for the relationship or balance between low and high frequency power (as well as cross-frequency coupling) with regard to the integration of stimuli of different durations (See 2.2.2. for details). Again, a medium or average balance may provide the highest degree of integration, whereas abnormal shifting of the power balance towards either low (as in schizophrenia; see above) or high (as in mania;(Matteo, Benedetta, Huang, Amore, \& Northoff, 2015) ) frequencies may lead decreased integration (see Figure $7 \mathrm{a}$ and $\mathrm{b}$ ). These inverted U-shape curves provide a continuum in neuronal mechanisms between healthy and psychiatric subjects which can be seen as the very basis of the dimensional approach suggested in RDoC.

\section{Figure $7 a$ and $b$}

\subsection{Limitations and future directions}

The investigation of spontaneous activity in the human brain is a fairly new endeavour, meaning that many aspects of it have not yet been fully explored (Weinberger \& Radulescu, 2015). For instance, the investigation of low-frequency intrinsic activity through fMRI has focussed to a large degree on functional connectivity. This has given us detailed information about spatial relationships but is less informative about the temporal properties of the activity. This is beginning to change as temporal measurements begin to be applied to fMRI data, such as activity variability (e.g., (Garrett et al., 2013; Z. Huang et al., 2014a; Z. Huang et al., 2014b; Martino et al., 2016), entropy (e.g., (N. W. Duncan et al., 2015; Wang \& Krystal, 2014)), and dynamic measurements of functional connectivity (e.g., (Betzel, Fukushima, He, Zuo, \& Sporns, 2016; Mitra, Snyder, Blazey, \& Raichle, 2015)). These novel approaches remain to be applied in schizophrenia, however, leaving open an interesting area of investigation.

How intrinsic temporal structure may differ in different dependent on regional functionality is an 
area that is also garnering more attention. Recent investigations in (J. D. Murray et al., 2014) and humans (Hasson, Chen, \& Honey, 2015) suggest that different regions/networks exhibit different intrinsic time windows within which different stimuli can be integrated. For instance, these temporal receptive windows (TRF) (Hasson et al., 2015) are rather short in sensory regions, while they seem to be extremely long in midline regions (Honey et al., 2012; J. D. Murray et al., 2014). Given the abnormalities in these regions in schizophrenia, one may assume that their TRF's, due to the changes in the spontaneous activity's temporal structure, may be altered which in turn predisposes abnormal processing of both internal and external stimuli. This remains to be explored though.

In addition to these spatial and temporal measures, the question of how the spontaneous activity interacts with stimuli remains unclear. Initial investigations suggested a linear or additive interaction between spontaneous activity and the stimulus, with the latter's activity changes superposing upon the former (Fox, Corbetta, Snyder, Vincent, \& Raichle, 2006; Northoff, Duncan, $\&$ Hayes, 2010). More recent investigations have, however, suggested that are non-additive interactions between spontaneous activity and stimuli, which may be driven by the pre-stimulus phase cycles of infraslow frequency fluctuations (see (He, 2013; Z Huang et al., 2015)). The spontaneous activity thus seems to exert influence upon the degree of neural activity changes a stimulus or task can evoke. One would expect that the changes in spontaneous activity observed in schizophrenia should then also impact the response to stimuli and subsequently stimulus-induced activity. One would therefore expect abnormal non-additive rest-stimulus interaction in schizophrenia, in turn contributing to specific symptoms. This remains to be investigated though.

The impact of especially the pre-stimulus resting state on subsequent stimulus-induced activity has also been framed in terms of predictive coding (Friston, 2010). Briefly, predictive coding postulates that stimulus-induced activity results from the comparison between the predicted input (as generated prior to the stimulus) and the actual input (the stimulus). The larger their discrepancy, i.e., the prediction error, the larger the amplitude of subsequent stimulus-induced activity. Abnormalities in predictive coding and specifically in generating the predicted input have been supposed to underlie delusions and auditory hallucinations (see for instance (Adams, Stephan, Brown, Frith, \& Friston, 2013; P. Corlett, Taylor, Wang, Fletcher, \& Krystal, 2010; P. R. Corlett, Honey, Krystal, \& Fletcher, 2011; Fletcher \& Frith, 2009; Fogelson, Litvak, Peled, Fernandez-del-Olmo, \& Friston, 2014; Judith M Ford et al., 2014; Horga, Schatz, Abi-Dargham, \& Peterson, 2014; Jardri \& Denève, 2013; Notredame, Pins, Deneve, \& Jardri, 2014). Without going into detail (see (Northoff, 2014a)), 
we would assume that the abnormalities in the predicted input can be traced to abnormal spatiotemporal structuring of the underlying spontaneous activity.

In this work, our account of psychopathological symptoms was limited to basic symptoms, cognitive changes, formal thought disorder, time perception, ego-disturbances, and body perception. This leaves out many other symptoms including auditory hallucinations, blunted affect, and delusions, to name but a few. As such, future research into how these symptoms are related to the spatiotemporal structure of spontaneous activity would be warranted. Moreover, we did not consider the different stages and subtypes of schizophrenia (e.g., early psychosis, paranoid schizophrenia, chronic schizophrenia, etc.). One would assume that any spatiotemporal abnormalities will differ between these different states, leading to the different symptom profiles. This represents another fruitful future research direction.

Finally, we have not discussed the biochemical bases of schizophrenia. Extensive research has linked the condition to alterations in the dopamine, glutamate and GABA transmitter systems, along with others (see for instance (Falkenberg, Westerhausen, Specht, \& Hugdahl, 2012; Hasan, Falkai, \& Wobrock, 2013; O. Howes, McCutcheon, \& Stone, 2015; O. D. Howes \& Murray, 2014; Daniel C Javitt, 2010; Moghaddam \& Javitt, 2012; R. M. Murray, Paparelli, Morrison, Marconi, \& Di Forti, 2013; Poels et al., 2014) . These systems have in turn been related to the spatiotemporal properties of spontaneous activity (see, for example, (N. W. Duncan, C. Wiebking, Z. MunozTorres, et al., 2014; N. W. Duncan, C. Wiebking, \& G. Northoff, 2014; Parker, Kumar, Lonergan, McMullan, \& Goodchild, 2015). How these factors may coincide in schizophrenia remains unclear at this point, however. Investigating this area may be of particular importance as it may point towards improved pharmacological treatment approaches for the condition.

\subsection{Conclusion}

To conclude, we have here reviewed the abnormalities in spontaneous activity in schizophrenia and linked them to specific psychopoathological symptoms. We fit these alterations into an approach to psychiatric disorders that takes alterations in the spatial and temporal properties of the brain as primary, which we term spatiotemporal psychopathology. This attempts to explain the symptoms of schizophrenia by starting from altered spatiotemporal properties of neural activity, which in turn cause abnormal experiences of space and time in the individual, and which finally manifest in the symptoms observed in the disease. We consequently suggest that the spatiotemporal structure of spontaneous activity provides the bridge between the neural and psychopathological levels. 
Finally, the detailed exploration of abnormal spatiotemporal features in both spontaneous activity and perception/experience could open the door for novel therapeutic interventions. Techniques like transcranial magnetic stimulation (TMS), transcranial direct current stimulation (tDCS), and deep brain stimulation (DBS) (see for instance(Krawinkel, Engel, \& Hummel, 2015) may be used in a spatiotemporally targeted way once the exact spatiotemporal mechanisms of specific symptoms are clear. Moreover, one may want to develop novel psychological treatment strategies such as training in time perception, as it has been done in healthy subjects ((Kwakye, Foss-Feig, Cascio, Stone, \& Wallace, 2010; Stevenson et al., 2014), or music therapy (Müller, Haffelder, Schlotmann, Schaefers, \& Teuchert-Noodt, 2014) that could ideally be tuned to an individual's specific neural activity properties.

\section{Acknowledgements}

The authors thank Wendy Carter for her assistance with the manuscript and for helpful suggestions on earlier drafts. GN received support from the Michael Smith Foundation (200809EJL-194083EJL-CECA-179644), the National Science Foundation of China (NSF No 31271195), and the Canadian Institutes of Health Research (201103MOP-244752- BSB-CECA-179644, 201103CCI248496-CCI-CECA-179644). NWD received support from the National Science Foundation of China (NSF31471072), the Taiwan Ministry of Science and Technology (105-2410-H-038-006MY3, 105-2410-H-038-005-MY2), and from Taipei Medical University (104-6402-006-110). The authors declare no competing interests.

\section{Figure captions}

\section{Figure captions}

Figure 1: Spatiotemporal psychopathology

Alterations in the brain's spontaneous activity (lower section) manifest themselves in altered spatial and temporal features (middle section). These may lead to an altered construction of time and space, e.g., spatiotemporal structure, by the brain's spontaneous activity which,in turn, impacts subsequent cognitive, sensory, motor, social and affective functions leading to the respective psychopathological symptoms (upper section).

Figure 2: Temporal structure in the brain's spontaneous activity

A) Neural activity in the brain can be separated into oscillations and fluctuations within different 
frequency bands. These range from high frequency gamma oscillations (approximately $25-100 \mathrm{~Hz}$ ) down to infra-slow fluctuations $(0.001-1 \mathrm{~Hz})$. These different frequencies are generally measured with EEG or MEG for the slow through high (delta to gamma), and with fMRI for infra-slow. B) Activity within the different freuquency bands is related to the activity in other bands. This cross-frequency coupling can take different forms. Here we illustrate phase to phase coupling, where the phase of the high frequency oscillation is coupled to the phase of the low frequency one. Other forms of coupling include power to power, and phase to power.

Figure 3: Linking temporal changes in the brain's spontaneous activity to psychopathological symptoms

A) Changes in the temporal structure of spontaneous activity in schizophrenia may underlie the symptoms of the condition. Here we illustrate how a shift to low-frequency activity and a disruption in cross-frequency coupling may result in symptoms such as disrupted cross-modal integration and other abnormalities in temporal perception.

B) The windows in which stimuli may be experienced as simultaneous may be increased in schizophrenia as a result in a shift from higher-frequency activity to low-frequency.

C) Reductions in slow-to-high frequency cross-frequency coupling may lead to problems in perceiving events as temporally distinct and in the correct order in schizophrenia.

Figure 4: Global temporal fragmentation in the spontaneous activity and generalized cognitive deficits

A schematic representation of the proposed link between temporal changes in the structure of spontaneous activity (lower section), disturbances in the perception/experience of time (middle section), and psychopathological symptoms in the cognitive, affective, sensorimotor, and social domains (upper section).

Figure 5: Altered network balance between the DMN and CEN and thought disorder in schizophrenia

A) An illustration of how altered spontaneous activity in schizophrenia (lower section) can lead to the observed change in the relationship between the DMN and CEN, where a positive inter-network correlation, rather than a negative one, is seen (middle section). This may then lead to a mixing of internally and externally oriented thoughts (upper section).

B) This switch from a negative to positive relationship, and the resulting mixing of thought contents, may then lead to symptoms such as thought insertion and withdrawal. 
Figure 6: Spatial alterations in the spontaneous activity and ego-disturbances

A schematic representation of the proposed link between changes in the spatial relationships within the spontaneous activity (lower section), mixing between internally and externally oriented thoughts (middle section), and psychopathological symptoms (upper section).

Figure 7: Dimensions of activity properties and psychopathology symptoms

A) The correlation between the DMN and CEN may support the correct segregation between internal and external mental contents at an optimal balance point. Deviation to either greater positive or negative correlation may then lead to pathological segregation, manifesting as depression or schizophrenia symptoms.

B) Similarly, an optimal balance between high and low frequency power may support appropriate integration of different stimuli. Shifts to either the high or low frequency ranges may result in abnormal integration and the symptoms of schizophrenia and mania.

\section{References:}

Adams, R. A., Stephan, K. E., Brown, H. R., Frith, C. D., \& Friston, K. J. (2013). The computational anatomy of psychosis. Front Psychiatry, 4, 47. doi: 10.3389/fpsyt.2013.00047

Alderson-Day, B., Diederen, K., Fernyhough, C., Ford, J. M., Horga, G., Margulies, D. S., . . . Turner, J. (2016). Auditory Hallucinations and the Brain's Resting-State Networks: Findings and Methodological Observations. Schizophr Bull, sbw078.

Allen, M., Smallwood, J., Christensen, J., Gramm, D., Rasmussen, B., Jensen, C. G., . . Lutz, A. (2013). The balanced mind: the variability of task-unrelated thoughts predicts error monitoring. Front Hum Neurosci, 7.

Allen, P., Modinos, G., Hubl, D., Shields, G., Cachia, A., Jardri, R., . . . Plaze, M. (2012). Neuroimaging auditory hallucinations in schizophrenia: from neuroanatomy to neurochemistry and beyond. Schizophr Bull, sbs066.

Alonso-Solís, A., Vives-Gilabert, Y., Grasa, E., Portella, M. J., Rabella, M., Sauras, R. B., . . . Pérez, V. (2015). Resting-state functional connectivity alterations in the default network of schizophrenia patients with persistent auditory verbal hallucinations. Schizophr Res, 161(2), 261-268.

Andreasen, N. C. (1999). A unitary model of schizophrenia: Bleuler's fragmented phrene as schizencephaly. Arch Gen Psychiatry, 56(9), 781-787.

Andreasen, N. C., Nopoulos, P., O’Leary, D. S., Miller, D. D., Wassink, T., \& Flaum, M. (1999). Defining the phenotype of schizophrenia: cognitive dysmetria and its neural mechanisms. Biol Psychiatry, 46(7), 908-920. 
Andreou, C., Nolte, G., Leicht, G., Polomac, N., Hanganu-Opatz, I. L., Lambert, M., . . Mulert, C. (2015). Increased resting-state gamma-band connectivity in first-episode schizophrenia. Schizophr Bull, 41(4), 930-939.

Angelopoulos, E., Koutsoukos, E., Maillis, A., Papadimitriou, G. N., \& Stefanis, C. (2014). Brain functional connectivity during the experience of thought blocks in schizophrenic patients with persistent auditory verbal hallucinations: An EEG study. Schizophr Res, 153(1), 109112.

Anticevic, A., Cole, M. W., Murray, J. D., Corlett, P. R., Wang, X.-J., \& Krystal, J. H. (2012). The role of default network deactivation in cognition and disease. Trends Cogn Sci, 16(12), 584592.

Anticevic, A., Cole, M. W., Repovs, G., Murray, J. D., Brumbaugh, M. S., Winkler, A. M., . . . Glahn, D. C. (2014). Characterizing thalamo-cortical disturbances in schizophrenia and bipolar illness. Cereb Cortex, 24(12), 3116-3130.

Aru, J., Aru, J., Priesemann, V., Wibral, M., Lana, L., Pipa, G., . . Vicente, R. (2015). Untangling cross-frequency coupling in neuroscience. Curr Opin Neurobiol, 31, 51-61.

Axmacher, N., Henseler, M. M., Jensen, O., Weinreich, I., Elger, C. E., \& Fell, J. (2010). Crossfrequency coupling supports multi-item working memory in the human hippocampus. Proceedings of the National Academy of Sciences, 107(7), 3228-3233.

Bai, Y., Nakao, T., Xu, J., Qin, P., Chaves, P., Heinzel, A., . . Tsai, S.-Y. (2015). Resting state glutamate predicts elevated pre-stimulus alpha during self-relatedness: A combined EEGMRS study on "rest-self overlap". Social neuroscience, 1-15.

Barch, D. M., \& Ceaser, A. (2012). Cognition in schizophrenia: core psychological and neural mechanisms. Trends Cogn Sci, 16(1), 27-34.

Barch, D. M., \& Sheffield, J. M. (2014). Cognitive impairments in psychotic disorders: common mechanisms and measurement. World Psychiatry, 13(3), 224-232.

Berman, R. A., Gotts, S. J., McAdams, H. M., Greenstein, D., Lalonde, F., Clasen, L., . . . Raznahan, A. (2015). Disrupted sensorimotor and social-cognitive networks underlie symptoms in childhood-onset schizophrenia. Brain, awv306.

Bernard, J. A., \& Mittal, V. A. (2014a). Cerebellar-motor dysfunction in schizophrenia and psychosis-risk: the importance of regional cerebellar analysis approaches. Front Psychiatry, 5(160.10), 3389.

Bernard, J. A., \& Mittal, V. A. (2014b). Dysfunctional Activation of the Cerebellum in Schizophrenia A Functional Neuroimaging Meta-Analysis. Clinical Psychological Science, 2167702614542463.

Betzel, R. F., Fukushima, M., He, Y., Zuo, X.-N., \& Sporns, O. (2016). Dynamic fluctuations coincide with periods of high and low modularity in resting-state functional brain networks. Neuroimage, 127, 287-297.

Bleuler, E. (1911). [Dementia praecox or the group of schizophrenias]. New York: International Universities Press.

Bleuler, E., \& Brill, A. A. (1924). Textbook of psychiatry.

Bluhm, R. L., Miller, J., Lanius, R. A., Osuch, E. A., Boksman, K., Neufeld, R., . . . Williamson, P. (2007). Spontaneous low-frequency fluctuations in the BOLD signal in schizophrenic patients: anomalies in the default network. Schizophr Bull, 33(4), 1004-1012.

Bullmore, E., \& Sporns, O. (2009). Complex brain networks: graph theoretical analysis of structural and functional systems. Nature Reviews Neuroscience, 10(3), 186-198.

Buzsaki, G. (2006). Rhythms of the brain Oxford University Press.

Capa, R. L., Duval, C. Z., Blaison, D., \& Giersch, A. (2014). Patients with schizophrenia selectively impaired in temporal order judgments. Schizophr Res, 156(1), 51-55. 
Carhart-Harris, R. L., Leech, R., Erritzoe, D., Williams, T. M., Stone, J. M., Evans, J., . . Nutt, D. J. (2013). Functional connectivity measures after psilocybin inform a novel hypothesis of early psychosis. Schizophr Bull, 39(6), 1343-1351.

Chen, A. C., Oathes, D. J., Chang, C., Bradley, T., Zhou, Z.-W., Williams, L. M., . . Etkin, A. (2013). Causal interactions between fronto-parietal central executive and default-mode networks in humans. Proceedings of the National Academy of Sciences, 110(49), 1994419949.

Chen, L., Vu, A., Xu, J., Moeller, S., Ugurbil, K., Yacoub, E., \& Feinberg, D. (2015). Evaluation of highly accelerated simultaneous multi-slice EPI for fMRI. Neuroimage, 104, 452-459.

Corbetta, M., Patel, G., \& Shulman, G. L. (2008). The reorienting system of the human brain: from environment to theory of mind. Neuron, 58(3), 306-324.

Corbetta, M., \& Shulman, G. L. (2011). Spatial neglect and attention networks. Annu Rev Neurosci, 34, 569.

Corlett, P., Taylor, J., Wang, X.-J., Fletcher, P., \& Krystal, J. (2010). Toward a neurobiology of delusions. Prog Neurobiol, 92(3), 345-369.

Corlett, P. R., Honey, G. D., Krystal, J. H., \& Fletcher, P. C. (2011). Glutamatergic model psychoses: prediction error, learning, and inference. Neuropsychopharmacology, 36(1), 294315.

Craig, A. (2009b). How do you feel-now? the anterior insula and human awareness. Nature Reviews Neuroscience, 10(1).

Ćurčić-Blake, B., van der Meer, L., Pijnenborg, G. H., David, A. S., \& Aleman, A. (2015). Insight and psychosis: Functional and anatomical brain connectivity and self-reflection in Schizophrenia. Hum Brain Mapp, 36(12), 4859-4868.

Cuthbert, B. N., \& Insel, T. R. (2013). Toward the future of psychiatric diagnosis: the seven pillars of RDoC. BMC Med, 11, 126. doi: 10.1186/1741-7015-11-126

D'Argembeau, A., Collette, F., Van der, L. M., Laureys, S., Del Fiore, G., Degueldre, C., . . . Salmon, E. (2005). Self-referential reflective activity and its relationship with rest: a PET study. Neuroimage., 25(2), 616-624.

Damaraju, E., Allen, E., Belger, A., Ford, J., McEwen, S., Mathalon, D., . . Preda, A. (2014). Dynamic functional connectivity analysis reveals transient states of dysconnectivity in schizophrenia. NeuroImage: Clinical, 5, 298-308.

Diez, A., Suazo, V., Casado, P., Martin-Loeches, M., \& Molina, V. (2013). Spatial distribution and cognitive correlates of gamma noise power in schizophrenia. Psychol Med, 43(06), 11751185 .

Dixon, N. F., \& Spitz, L. (1980). The detection of auditory visual desynchrony. Perception, 9(6), 719-721.

Duncan, N., Wiebking, C., Zhang, J., Huang, Z., \& Northoff, G. (2014). GABA -A receptor binding and neuronal variability

Duncan, N. W., Hayes, D. J., Wiebking, C., Brice, T., Pietruska, K., Chen, D., . . Northoff, G. (2015). Negative childhood experiences alter a prefrontal-insular-motor cortical network in healthy adults: A multimodal rsfMRI-fMRI-MRS-dMRI study. Hum Brain Mapp, in press.

Duncan, N. W., Wiebking, C., Munoz-Torres, Z., \& Northoff, G. (2014). How to investigate neurobiochemical relationships on a regional level in humans? Methodological considerations for combining functional with biochemical imaging. J Neurosci Methods, 221, 183-188.

Duncan, N. W., Wiebking, C., \& Northoff, G. (2014). Associations of regional GABA and glutamate with intrinsic and extrinsic neural activity in humans-A review of multimodal imaging studies. Neurosci Biobehav Rev, 47C, 36-52. doi: 10.1016/j.neubiorev.2014.07.016

Ebisch, S. J., \& Aleman, A. (2016). The fragmented self: imbalance between intrinsic and extrinsic self-networks in psychotic disorders. The Lancet Psychiatry. 
Falkenberg, L. E., Westerhausen, R., Specht, K., \& Hugdahl, K. (2012). Resting-state glutamate level in the anterior cingulate predicts blood-oxygen level-dependent response to cognitive control. Proceedings of the National Academy of Sciences, 109(13), 5069-5073.

Fatemi, S. H., \& Folsom, T. D. (2009). The neurodevelopmental hypothesis of schizophrenia, revisited. Schizophr Bull, sbn187.

Fletcher, P. C., \& Frith, C. D. (2009). Perceiving is believing: a Bayesian approach to explaining the positive symptoms of schizophrenia. Nature Reviews Neuroscience, 10(1), 48-58.

Fogelson, N., Litvak, V., Peled, A., Fernandez-del-Olmo, M., \& Friston, K. (2014). The functional anatomy of schizophrenia: A dynamic causal modeling study of predictive coding. Schizophr Res, 158(1), 204-212.

Ford, J. M., Dierks, T., Fisher, D. J., Herrmann, C. S., Hubl, D., Kindler, J., . . van Lutterveld, R. (2012). Neurophysiological studies of auditory verbal hallucinations. Schizophr Bull, 38(4), 715-723. doi: 10.1093/schbul/sbs009

Ford, J. M., Morris, S. E., Hoffman, R. E., Sommer, I., Waters, F., McCarthy-Jones, S., . . . Badcock, J. C. (2014). Studying hallucinations within the NIMH RDoC framework. Schizophr Bull, 40(Suppl 4), S295-S304.

Fox, M. D., Corbetta, M., Snyder, A. Z., Vincent, J. L., \& Raichle, M. E. (2006). Spontaneous neuronal activity distinguishes human dorsal and ventral attention systems. Proceedings of the National Academy of Sciences, 103(26), 10046-10051.

Friston, K. (2010). The free-energy principle: a unified brain theory? Nature Reviews Neuroscience, 11(2), 127-138.

Frith, C. D. (1992). The cognitive neuropsychology of schizophrenia: Psychology Press.

Fryer, S. L., Roach, B. J., Ford, J. M., Turner, J. A., Van Erp, T. G., Voyvodic, J., . . O'Leary, D. (2015). Relating intrinsic low-frequency bold cortical oscillations to cognition in schizophrenia. Neuropsychopharmacology, 40(12), 2705-2714.

Fuchs, T. (2007). The temporal structure of intentionality and its disturbance in schizophrenia. Psychopathology, 40(4), 229-235.

Fuchs, T. (2009). Das Gehirn-ein Beziehungsorgan: eine phänomenologisch-ökologische Konzeption: W. Kohlhammer Verlag.

Fuchs, T. (2013). Temporality and psychopathology. Phenomenology and the cognitive sciences, 12(1), 75-104.

Gallinat, J., McMahon, K., Kühn, S., Schubert, F., \& Schaefer, M. (2015). Cross-sectional Study of Glutamate in the Anterior Cingulate and Hippocampus in Schizophrenia. Schizophr Bull, sbv124.

Garakh, Z., Zaytseva, Y., Kapranova, A., Fiala, O., Horacek, J., Shmukler, A., . . Strelets, V. B. (2015). EEG correlates of a mental arithmetic task in patients with first episode schizophrenia and schizoaffective disorder. Clinical Neurophysiology, 126(11), 2090-2098.

Garrett, D. D., Samanez-Larkin, G. R., MacDonald, S. W. S., Lindenberger, U., McIntosh, A. R., \& Grady, C. L. (2013). Moment-to-moment brain signal variability: A next frontier in human brain mapping? Neuroscience \& Biobehavioral Reviews, 37(4), 610-624. doi: http://dx.doi.org/10.1016/j.neubiorev.2013.02.015

Garrity, A. G., Pearlson, G. D., McKiernan, K., Lloyd, D., Kiehl, K. A., \& Calhoun, V. D. (2007). Aberrant "default mode" functional connectivity in schizophrenia. American Journal of Psychiatry.

Giersch, A., Lalanne, L., Van Assche, M., \& Elliott, M. A. (2013). On disturbed time continuity in schizophrenia: an elementary impairment in visual perception? Front Psychol, 4.

Goel, V., \& Dolan, R. J. (2003a). Explaining modulation of reasoning by belief. Cognition, 87(1), B11-22.

Goel, V., \& Dolan, R. J. (2003b). Reciprocal neural response within lateral and ventral medial prefrontal cortex during hot and cold reasoning. Neuroimage, 20(4), 2314-2321. 
Hanslmayr, S., Backes, H., Straub, S., Popov, T., Langguth, B., Hajak, G., . . L Landgrebe, M. (2013). Enhanced resting-state oscillations in schizophrenia are associated with decreased synchronization during inattentional blindness. Hum Brain Mapp, 34(9), 2266-2275. doi: 10.1002/hbm.22064

Hasan, A., Falkai, P., \& Wobrock, T. (2013). Transcranial brain stimulation in schizophrenia: targeting cortical excitability, connectivity and plasticity. Current medicinal chemistry, 20(3), 405-413.

Hasson, U., Chen, J., \& Honey, C. J. (2015). Hierarchical process memory: memory as an integral component of information processing. Trends Cogn Sci, 19(6), 304-313.

He, B. J. (2013). Spontaneous and task-evoked brain activity negatively interact. The Journal of Neuroscience, 33(11), 4672-4682.

He, B. J., \& Zempel, J. M. (2013). Average is optimal: an inverted-U relationship between trial-totrial brain activity and behavioral performance. PLoS Comput Biol, 9(11), e1003348.

Hiltunen, T., Kantola, J., Elseoud, A. A., Lepola, P., Suominen, K., Starck, T., . . Palva, S. (2014). Infra-slow EEG fluctuations are correlated with resting-state network dynamics in fMRI. The Journal of Neuroscience, 34(2), 356-362.

Hirano, Y., Oribe, N., Kanba, S., Onitsuka, T., Nestor, P. G., \& Spencer, K. M. (2015). Spontaneous Gamma Activity in Schizophrenia. JAMA psychiatry.

Hoffman, R. E. (2007). A social deafferentation hypothesis for induction of active schizophrenia. Schizophr Bull, 33(5), 1066-1070.

Holt, D. J., Cassidy, B. S., Andrews-Hanna, J. R., Lee, S. M., Coombs, G., Goff, D. C., .. . Moran, J. M. (2011). An anterior-to-posterior shift in midline cortical activity in schizophrenia during self-reflection. Biol.Psychiatry, 69(5), 415-423.

Honey, C. J., Thesen, T., Donner, T. H., Silbert, L. J., Carlson, C. E., Devinsky, O., . . Hasson, U. (2012). Slow cortical dynamics and the accumulation of information over long timescales. Neuron, 76(2), 423-434.

Hong, L. E., Turano, K. A., O’Neill, H., Hao, L., Wonodi, I., McMahon, R. P., . . Thaker, G. K. (2008). Refining the predictive pursuit endophenotype in schizophrenia. Biol Psychiatry, 63(5), 458-464.

Horga, G., Schatz, K. C., Abi-Dargham, A., \& Peterson, B. S. (2014). Deficits in predictive coding underlie hallucinations in schizophrenia. The Journal of Neuroscience, 34(24), 8072-8082.

Howes, O., McCutcheon, R., \& Stone, J. (2015). Glutamate and dopamine in schizophrenia: An update for the 21st century. Journal of Psychopharmacology, 0269881114563634.

Howes, O. D., \& Murray, R. M. (2014). Schizophrenia: an integrated sociodevelopmental-cognitive model. The Lancet, 383(9929), 1677-1687.

Huang, Z., Dai, R., Wu, X., Yang, Z., Liu, D., Hu, J., . . Northoff, G. (2014a). The self and its resting state in consciousness: an investigation of the vegetative state. Hum Brain Mapp, 35(5), 1997-2008. doi: 10.1002/hbm.22308

Huang, Z., Ferri, F., Longtin, A., Dumont, G., \& Northoff, G. (2016). Is there non-additive interaction between resting state and stimulus-induced activity? Phase dependency and the spontaneous activity's temporal structure. Cereb Cortex.

Huang, Z., Obara, N., Davis IV, H. H., Pokorny, J., \& Northoff, G. (2016). The temporal structure of resting-state brain activity in the medial prefrontal cortex predicts self-consciousness. Neuropsychologia.

Huang, Z., Wang, Z., Zhang, J., Dai, R., Wu, J., Li, Y., . . Northoff, G. (2014b). Altered temporal variance and neural synchronization of spontaneous brain activity in anesthesia. Hum Brain Mapp. doi: 10.1002/hbm.22556

Huang, Z., Zhang, J., Wu, J., Qin, P., Wu, X., Wang, Z., . . Northoff, G. (2015). Decoupled temporal variability and signal synchronization of spontaneous brain activity in loss of consciousness: an fMRI study in anethesia. Neuroimage. 
Iwabuchi, S. J., Liddle, P. F., \& Palaniyappan, L. (2015). Structural connectivity of the salienceexecutive loop in schizophrenia. Eur Arch Psychiatry Clin Neurosci, 265(2), 163-166.

Jardri, R., \& Denève, S. (2013). Circular inferences in schizophrenia. Brain, 136(11), 3227-3241.

Javitt, D. C. (2009). Sensory processing in schizophrenia: neither simple nor intact. Schizophr Bull, 35(6), 1059-1064. doi: 10.1093/schbul/sbp110

Javitt, D. C. (2010). Glutamatergic theories of schizophrenia. Israel Journal of Psychiatry and Related Sciences, 47(1), 4.

Javitt, D. C., \& Freedman, R. (2014). Sensory Processing Dysfunction in the Personal Experience and Neuronal Machinery of Schizophrenia.

Javitt, D. C., \& Sweet, R. A. (2015). Auditory dysfunction in schizophrenia: integrating clinical and basic features. Nature Reviews Neuroscience, 16(9), 535-550.

Kam, J. W., Bolbecker, A. R., O'Donnell, B. F., Hetrick, W. P., \& Brenner, C. A. (2013). Resting state EEG power and coherence abnormalities in bipolar disorder and schizophrenia. $J$ Psychiatr Res, 47(12), 1893-1901. doi: 10.1016/j.jpsychires.2013.09.009

Kantrowitz, J. T., Hoptman, M. J., Leitman, D. I., Moreno-Ortega, M., Lehrfeld, J. M., Dias, E., . . . Javitt, D. C. (2015). Neural Substrates of Auditory Emotion Recognition Deficits in Schizophrenia. The Journal of Neuroscience, 35(44), 14909-14921.

Karbasforoushan, H., \& Woodward, N. (2012). Resting-state networks in schizophrenia. Curr Top Med Chem, 12(21), 2404-2414.

Kaufmann, T., Skåtun, K. C., Alnæs, D., Doan, N. T., Duff, E. P., Tønnesen, S., . . Lagerberg, T. V. (2015). Disintegration of sensorimotor brain networks in schizophrenia. Schizophr Bull, 41(6), 1326-1335.

Kean, C. (2009). Silencing the self: schizophrenia as a self-disturbance. Schizophr Bull, sbp043.

Kikuchi, M., Hashimoto, T., Nagasawa, T., Hirosawa, T., Minabe, Y., Yoshimura, M., ... Koenig, T. (2011). Frontal areas contribute to reduced global coordination of resting-state gamma activities in drug-naive patients with schizophrenia. Schizophr Res, 130(1-3), 187-194. doi: 10.1016/j.schres.2011.06.003

Kim, J. S., Shin, K. S., Jung, W. H., Kim, S. N., Kwon, J. S., \& Chung, C. K. (2014). Power spectral aspects of the default mode network in schizophrenia: an MEG study. $B M C$ Neurosci, 15(1), 1.

Kindler, J., Jann, K., Homan, P., Hauf, M., Walther, S., Strik, W., . . Hubl, D. (2013). Static and dynamic characteristics of cerebral blood flow during the resting state in schizophrenia. Schizophr Bull, sbt180.

Kirihara, K., Rissling, A. J., Swerdlow, N. R., Braff, D. L., \& Light, G. A. (2012). Hierarchical organization of gamma and theta oscillatory dynamics in schizophrenia. Biol Psychiatry, 71(10), 873-880. doi: 10.1016/j.biopsych.2012.01.016

Klosterkötter, J., Ebel, H., Schultze-Lutter, F., \& Steinmeyer, E. M. (1996). Diagnostic validity of basic symptoms. Eur Arch Psychiatry Clin Neurosci, 246(3), 147-154.

Klosterkötter, J., Hellmich, M., Steinmeyer, E. M., \& Schultze-Lutter, F. (2001). Diagnosing schizophrenia in the initial prodromal phase. Arch Gen Psychiatry, 58(2), 158-164.

Kraepelin, E., \& Diefendorf, A. R. (1915). Clinical psychiatry: a text-book for students and physicians: Macmillan.

Krawinkel, L. A., Engel, A. K., \& Hummel, F. C. (2015). Modulating pathological oscillations by rhythmic non-invasive brain stimulation-a therapeutic concept? Front Syst Neurosci, 9.

Kwakye, L. D., Foss-Feig, J. H., Cascio, C. J., Stone, W. L., \& Wallace, M. T. (2010). Altered auditory and multisensory temporal processing in autism spectrum disorders. Front Integr Neurosci, 4.

Lakatos, P., Schroeder, C. E., Leitman, D. I., \& Javitt, D. C. (2013). Predictive suppression of cortical excitability and its deficit in schizophrenia. The Journal of Neuroscience, 33(28), 11692-11702. 
Leicht, G., Vauth, S., Polomac, N., Andreou, C., Rauh, J., Mußmann, M., . . . Mulert, C. (2016). EEG-Informed fMRI Reveals a Disturbed Gamma-Band-Specific Network in Subjects at High Risk for Psychosis. Schizophr Bull, 42(1), 239-249.

Leube, D., Whitney, C., \& Kircher, T. (2008). The neural correlates of ego-disturbances (passivity phenomena) and formal thought disorder in schizophrenia. Eur Arch Psychiatry Clin Neurosci, 258(5), 22-27.

Lewis, D. A., Curley, A. A., Glausier, J. R., \& Volk, D. W. (2012). Cortical parvalbumin interneurons and cognitive dysfunction in schizophrenia. Trends Neurosci, 35(1), 57-67.

Li, M., Deng, W., He, Z., Wang, Q., Huang, C., Jiang, L., . . Ma, X. (2015). A splitting brain: Imbalanced neural networks in schizophrenia. Psychiatry Research: Neuroimaging, 232(2), 145-153.

Lipsman, N., Nakao, T., Kanayama, N., Krauss, J. K., Anderson, A., Giacobbe, P., . . Northoff, D. G. (2014). Neural overlap between resting state and self-relevant activity in human subcallosal cingulate cortex - Single unit recording in an intracranial study. Cortex-in press(0). doi: http://dx.doi.org/10.1016/j.cortex.2014.09.008

Littow, H., Huossa, V., Karjalainen, S., Jääskeläinen, E., Haapea, M., Miettunen, J., . . Veijola, J. (2015). Aberrant functional connectivity in the default mode and central executive networks in subjects with schizophrenia-a whole-brain resting-state ICA study. Front Psychiatry, 6.

Liu, H., Kaneko, Y., Ouyang, X., Li, L., Hao, Y., Chen, E. Y., . . . Liu, Z. (2012). Schizophrenic patients and their unaffected siblings share increased resting-state connectivity in the tasknegative network but not its anticorrelated task-positive network. Schizophr Bull, 38(2), 285-294. doi: 10.1093/schbul/sbq074

Liu, J., Corbera, S., \& Wexler, B. E. (2014). Neural activation abnormalities during self-referential processing in schizophrenia: an fMRI study. Psychiatry Research: Neuroimaging, 222(3), $165-171$.

Lo, C. Y., Su, T. W., Huang, C. C., Hung, C. C., Chen, W. L., Lan, T. H., . . Bullmore, E. T. (2015). Randomization and resilience of brain functional networks as systems-level endophenotypes of schizophrenia. Proc Natl Acad Sci U S A, 112(29), 9123-9128. doi: 10.1073/pnas.1502052112

Logothetis, N. K., Murayama, Y., Augath, M., Steffen, T., Werner, J., \& Oeltermann, A. (2009). How not to study spontaneous activity. Neuroimage, 45(4), 1080-1089. doi: 10.1016/j.neuroimage.2009.01.010

Logothetis, N. K., Pauls, J., Augath, M., Trinath, T., \& Oeltermann, A. (2001). Neurophysiological investigation of the basis of the fMRI signal. Nature, 412(6843), 150-157.

Manoliu, A., Riedl, V., Zherdin, A., Mühlau, M., Schwerthöffer, D., Scherr, M., . . Bäuml, J. (2014). Aberrant dependence of default mode/central executive network interactions on anterior insular salience network activity in schizophrenia. Schizophr Bull, 40(2), 428-437.

Martin, B., Giersch, A., Huron, C., \& van Wassenhove, V. (2013). Temporal event structure and timing in schizophrenia: preserved binding in a longer "now". Neuropsychologia, 51(2), 358-371. doi: 10.1016/j.neuropsychologia.2012.07.002

Martino, M., Magioncalda, P., Huang, Z., Conio, B., Piaggio, N., Duncan, N. W., .. . Wolff, A. (2016). Contrasting variability patterns in the default mode and sensorimotor networks balance in bipolar depression and mania. Proceedings of the National Academy of Sciences, 113(17), 4824-4829.

Matsuo, K., Chen, S.-H. A., Liu, C.-M., Liu, C.-C., Hwang, T.-J., Hsieh, M. H., . . Tseng, W.-Y. I. (2013). Stable signatures of schizophrenia in the cortical-subcortical-cerebellar network using fMRI of verbal working memory. Schizophr Res, 151(1), 133-140.

Matteo, M., Benedetta, P., Huang, Z., Amore, M., \& Northoff, G. (2015). Opposite variability pattern in default mode and sensorimotor netowrk in manic and depressive biopolar disorder. submitted. 
Meda, S. A., Wang, Z., Ivleva, E. I., Poudyal, G., Keshavan, M. S., Tamminga, C. A., . . Calhoun, V. D. (2015). Frequency-specific neural signatures of spontaneous low-frequency resting state fluctuations in psychosis: evidence from bipolar-schizophrenia network on intermediate phenotypes (B-SNIP) consortium. Schizophr Bull, sbv064.

Menon, M., Schmitz, T. W., Anderson, A. K., Graff, A., Korostil, M., Mamo, D., . . Kapur, S. (2011). Exploring the neural correlates of delusions of reference. Biol Psychiatry, 70(12), 1127-1133.

Mishara, A., Bonoldi, I., Allen, P., Rutigliano, G., Perez, J., Fusar-Poli, P., \& McGuire, P. (2015). Neurobiological Models of Self-Disorders in Early Schizophrenia. Schizophr Bull. doi: $10.1093 / \mathrm{schbul} / \mathrm{sbv} 123$

Mitra, A., Snyder, A. Z., Blazey, T., \& Raichle, M. E. (2015). Lag threads organize the brain's intrinsic activity. Proceedings of the National Academy of Sciences, 112(17), E2235-E2244.

Moghaddam, B., \& Javitt, D. (2012). From revolution to evolution: the glutamate hypothesis of schizophrenia and its implication for treatment. Neuropsychopharmacology, 37(1), 4-15.

Molina, V., Bachiller, A., Suazo, V., Lubeiro, A., Poza, J., \& Hornero, R. (2016). Noise power associated with decreased task-induced variability of brain electrical activity in schizophrenia. Eur Arch Psychiatry Clin Neurosci, 266(1), 55-61.

Monto, S., Palva, S., Voipio, J., \& Palva, J. M. (2008). Very slow EEG fluctuations predict the dynamics of stimulus detection and oscillation amplitudes in humans. J Neurosci, 28(33), 8268-8272. doi: 10.1523/JNEUROSCI.1910-08.2008

Moran, L. V., Tagamets, M. A., Sampath, H., O’Donnell, A., Stein, E. A., Kochunov, P., \& Hong, L. E. (2013). Disruption of anterior insula modulation of large-scale brain networks in schizophrenia. Biol Psychiatry, 74(6), 467-474.

Mouchlianitis, E., Bloomfield, M. A., Law, V., Beck, K., Selvaraj, S., Rasquinha, N., . . Stone, J. (2015). Treatment-Resistant Schizophrenia Patients Show Elevated Anterior Cingulate Cortex Glutamate Compared to Treatment-Responsive. Schizophr Bull, sbv151.

Müller, W., Haffelder, G., Schlotmann, A., Schaefers, A. T., \& Teuchert-Noodt, G. (2014). Amelioration of psychiatric symptoms through exposure to music individually adapted to brain rhythm disorders-a randomised clinical trial on the basis of fundamental research. Cognitive neuropsychiatry, 19(5), 399-413.

Murray, J. D., Bernacchia, A., Freedman, D. J., Romo, R., Wallis, J. D., Cai, X., . . Lee, D. (2014). A hierarchy of intrinsic timescales across primate cortex. Nat Neurosci.

Murray, R. M., Paparelli, A., Morrison, P. D., Marconi, A., \& Di Forti, M. (2013). What can we learn about schizophrenia from studying the human model, drug-induced psychosis? American Journal of Medical Genetics Part B: Neuropsychiatric Genetics, 162(7), 661-670.

Najas-García, A., Rufián, S., \& Rojo, E. (2014). Neurodevelopment or neurodegeneration: Review of theories of schizophrenia. Actas Esp Psiquiatr, 42(4), 185-195.

Nakao, T., Bai, Y., Nashiwa, H., \& Northoff, G. (2013). Resting-state EEG power predicts conflictrelated brain activity in internally guided but not in externally guided decision-making. Neuroimage, 66, 9-21. doi: 10.1016/j.neuroimage.2012.10.034

Nakao, T., Ohira, H., \& Northoff, G. (2012). Distinction between Externally vs. Internally Guided Decision-Making: Operational Differences, Meta-Analytical Comparisons and Their Theoretical Implications. Front Neurosci, 6, 31. doi: 10.3389/fnins.2012.00031

Narayanan, M., Huynh, J. L., Wang, K., Yang, X., Yoo, S., McElwee, J., . . X Xie, T. (2014). Common dysregulation network in the human prefrontal cortex underlies two neurodegenerative diseases. Molecular systems biology, 10(7).

Nekovarova, T., Fajnerova, I., Horacek, J., \& Spaniel, F. (2015). Bridging disparate symptoms of schizophrenia: a triple network dysfunction theory. Cognitive deficits in schizophrenia and other neuropsychiatric disorders: Convergence of preclinical and clinical evidence, 46. 
Nelson, B., Whitford, T., Lavoie, S., \& Sass, L. (2014a). What are the neurocognitive correlates of basic self-disturbance in schizophrenia?: Integrating phenomenology and neurocognition. Part 1 (Source monitoring deficits). Schizophr Res, 152(1), 12-19.

Nelson, B., Whitford, T., Lavoie, S., \& Sass, L. (2014b). What are the neurocognitive correlates of basic self-disturbance in schizophrenia?: Integrating phenomenology and neurocognition: Part 2 (Aberrant salience). Schizophr Res, 152(1), 20-27.

Northoff, G. (2002). What catatonia can tell us about "top-down modulation": a neuropsychiatric hypothesis. Behavioral and Brain Sciences, 25(05), 555-577.

Northoff, G. (2012). Immanuel Kant's mind and the brain's resting state. Trends Cogn Sci, 16(7), 356-359. doi: 10.1016/j.tics.2012.06.001

Northoff, G. (2014). How Is Our Self Altered in Psychiatric Disorders? A Neurophenomenal Approach to Psychopathological Symptoms. Psychopathology. doi: 10.1159/000363351

Northoff, G. (2014a). Unlocking the Brain: Volume 1: Coding (Vol. 1): Oxford University Press.

Northoff, G. (2014b). Unlocking the Brain. Volume II: Consciousness: Oxford: Oxford University Press.

Northoff, G. (2014c). Minding the Brain: A Guide to Philosophy \& Neuroscience: Palgrave Macmillan.

Northoff, G. (2015). Is the self a higher-order of fundamental funciton of the brain? The "basis model of self-specifity" and its encoding by the brain's spontaneous activity. Cognitive Neuroscience, Journal of.

Northoff, G. (2015a). Spatiotemporal Psychopathology I: Is depression a spatiotemporal disorder of the brain's resting state? Journal of Affective Disorder.

Northoff, G. (2016). How do resting state changes in depression translate into psychopathological symptoms? From 'Spatiotemporal correspondence'to 'Spatiotemporal Psychopathology'. Current opinion in psychiatry, 29(1), 18-24.

Northoff, G. (2016a). Neuroscience and Whitehead I: Neuro-ecological Model of Brain. Axiomathes, 1-34.

Northoff, G. (2016b). Neuroscience and Whitehead II: Process-Based Ontology of Brain. Axiomathes, 1-25.

Northoff, G., Duncan, N. W., \& Hayes, D. J. (2010). The brain and its resting state activity-experimental and methodological implications. Prog Neurobiol, 92(4), 593-600. doi: 10.1016/j.pneurobio.2010.09.002

Northoff, G., Heinzel, A., Bermpohl, F., Niese, R., Pfennig, A., Pascual-Leone, A., \& Schlaug, G. (2004). Reciprocal modulation and attenuation in the prefrontal cortex: an fMRI study on emotional-cognitive interaction. Hum Brain Mapp, 21(3), 202-212. doi: 10.1002/hbm.20002

Northoff, G., Kotter, R., Baumgart, F., Danos, P., Boeker, H., Kaulisch, T., . . Bogerts, B. (2004). Orbitofrontal cortical dysfunction in akinetic catatonia: a functional magnetic resonance imaging study during negative emotional stimulation. Schizophr Bull, 30(2), 405-427.

Northoff, G., \& Sibille, E. (2014). Cortical GABA neurons and self-focus in depression: a model linking cellular, biochemical and neural network findings. Mol Psychiatry, 19(9), 959-959.

Northoff, G., \& Sibille, E. (2014). Why are cortical GABA neurons relevant to internal focus in depression\&quest; A cross-level model linking cellular, biochemical and neural network findings. Mol Psychiatry, 19(9), 966-977.

Northoff, G., \& Stanghellini, G. (2016). How to link brain and experience? Spatiotemporal Psychopathology of the Lived Body. Front Hum Neurosci, 10, 172.

Notredame, C.-E., Pins, D., Deneve, S., \& Jardri, R. (2014). What visual illusions teach us about schizophrenia. Front Integr Neurosci, 8.

Orliac, F., Naveau, M., Joliot, M., Delcroix, N., Razafimandimby, A., Brazo, P., . . Delamillieure, P. (2013). Links among resting-state default-mode network, salience network, and symptomatology in schizophrenia. Schizophr Res, 148(1), 74-80. 
Palaniyappan, L., Simmonite, M., White, T. P., Liddle, E. B., \& Liddle, P. F. (2013). Neural primacy of the salience processing system in schizophrenia. Neuron, 79(4), 814-828.

Pankow, A., Knobel, A., Voss, M., \& Heinz, A. (2012). Neurobiological correlates of delusion: beyond the salience attribution hypothesis. Neuropsychobiology, 66(1), 33-43.

Panksepp, J. (2004). Textbook of biological psychiatry: Wiley Online Library.

Parker, L. M., Kumar, N. N., Lonergan, T., McMullan, S., \& Goodchild, A. K. (2015). Distribution and neurochemical characterization of neurons in the rat ventrolateral medulla activated by glucoprivation. Brain Structure and Function, 220(1), 117-134.

Parnas, J., Sass, L. A., \& Zahavi, D. (2012). Rediscovering psychopathology: the epistemology and phenomenology of the psychiatric object. Schizophr Bull, sbs 153.

Pinal, D., Zurrón, M., Díaz, F., \& Sauseng, P. (2015). Stuck in default mode: inefficient crossfrequency synchronization may lead to age-related short-term memory decline. Neurobiol Aging, 36(4), 1611-1618.

Poels, E. M., Kegeles, L. S., Kantrowitz, J. T., Javitt, D. C., Lieberman, J. A., Abi-Dargham, A., \& Girgis, R. R. (2014). Glutamatergic abnormalities in schizophrenia: a review of proton MRS findings. Schizophr Res, 152(2-3), 325-332. doi: 10.1016/j.schres.2013.12.013

Popov, T. G., Carolus, A., Schubring, D., Popova, P., Miller, G. A., \& Rockstroh, B. S. (2015). Targeted training modifies oscillatory brain activity in schizophrenia patients. NeuroImage: Clinical, 7, 807-814.

Qin, P., Grimm, S., Duncan, N. W., Fan, Y., Huang, Z., Lane, T., . . Northoff, G. (2016). Spontaneous activity in default-mode network predicts ascription of self-relatedness to stimuli. Soc Cogn Affect Neurosci, nsw008.

Qin, P., Liu, Y., Shi, J., Wang, Y., Duncan, N., Gong, Q., . . Northoff, G. (2012). Dissociation between anterior and posterior cortical regions during self-specificity and familiarity: a combined fMRI-meta-analytic study. Hum Brain Mapp, 33(1), 154-164. doi: $10.1002 / \mathrm{hbm} .21201$

Qin, P., \& Northoff, G. (2011). How is our self related to midline regions and the default-mode network? Neuroimage., 57(3), 1221-1233.

Radua, J., Schmidt, A., Borgwardt, S., Heinz, A., Schlagenhauf, F., McGuire, P., \& Fusar-Poli, P. (2015). Ventral Striatal Activation During Reward Processing in Psychosis: A Neurofunctional Meta-Analysis. JAMA psychiatry, 72(12), 1243-1251.

Raichle, M. E. (2009). A brief history of human brain mapping. Trends Neurosci, 32(2), 118-126.

Raichle, M. E. (2011). The restless brain. Brain Connect, 1(1), 3-12.

Raichle, M. E. (2015). The restless brain: how intrinsic activity organizes brain function. Philosophical Transactions of the Royal Society of London B: Biological Sciences, 370(1668), 20140172.

Ranlund, S., Nottage, J., Shaikh, M., Dutt, A., Constante, M., Walshe, M., . . Bramon, E. (2014). Resting EEG in psychosis and at-risk populations - A possible endophenotype? Schizophr Res, 153(1-3), 96-102. doi: 10.1016/j.schres.2013.12.017

Razavi, N., Jann, K., Koenig, T., Kottlow, M., Hauf, M., Strik, W., \& Dierks, T. (2013). Shifted coupling of EEG driving frequencies and fMRI resting state networks in schizophrenia spectrum disorders. PLoS One, 8(10), e76604. doi: 10.1371/journal.pone.0076604

Robinson, J. J. D., Wagner, N.-F., \& Northoff, G. (2015). Is the Sense of Agency in Schizophrenia Influenced by Resting-State Variation in Self-Referential Regions of the Brain? Schizophr Bull, sbv102.

Rotarska-Jagiela, A., van, d. V., V, Oertel-Knochel, V., Uhlhaas, P. J., Vogeley, K., \& Linden, D. E. (2010). Resting-state functional network correlates of psychotic symptoms in schizophrenia. Schizophr.Res, 117(1), 21-30.

Roux, F., \& Uhlhaas, P. J. (2014). Working memory and neural oscillations: alpha-gamma versus theta-gamma codes for distinct WM information? Trends Cogn Sci, 18(1), 16-25. 
Sadaghiani, S., Hesselmann, G., Friston, K. J., \& Kleinschmidt, A. (2010). The relation of ongoing brain activity, evoked neural responses, and cognition. Front Syst Neurosci, 4.

Sadaghiani, S., \& Kleinschmidt, A. (2013). Functional interactions between intrinsic brain activity and behavior. Neuroimage, 80(0), 379-386. doi: http://dx.doi.org/10.1016/j.neuroimage.2013.04.100

Scheeringa, R., Koopmans, P. J., van Mourik, T., Jensen, O., \& Norris, D. G. (2016). The relationship between oscillatory EEG activity and the laminar-specific BOLD signal. Proceedings of the National Academy of Sciences, 201522577.

Scheeringa, R., Mazaheri, A., Bojak, I., Norris, D. G., \& Kleinschmidt, A. (2011). Modulation of visually evoked cortical FMRI responses by phase of ongoing occipital alpha oscillations. The Journal of Neuroscience, 31(10), 3813-3820.

Schneider, F., Bermpohl, F., Heinzel, A., Rotte, M., Walter, M., Tempelmann, C., . . Northoff, G. (2008). The resting brain and our self: self-relatedness modulates resting state neural activity in cortical midline structures. Neuroscience, 157(1), 120-131.

Schneider, K. (1974). Psychopathology. Stuttgart: Thieme.

Schultze-Lutter, F. (2009). Subjective symptoms of schizophrenia in research and the clinic: the basic symptom concept. Schizophr Bull, 35(1), 5-8.

Seeley, W. W., Menon, V., Schatzberg, A. F., Keller, J., Glover, G. H., Kenna, H., . . Greicius, M. D. (2007). Dissociable intrinsic connectivity networks for salience processing and executive control. The Journal of Neuroscience, 27(9), 2349-2356.

Shad, M. U., Keshavan, M. S., Steinberg, J. L., Mihalakos, P., Thomas, B. P., Motes, M. A., . . Tamminga, C. A. (2012). Neurobiology of self-awareness in schizophrenia: an fMRI study. Schizophr Res, 138(2-3), 113-119. doi: 10.1016/j.schres.2012.03.016

Sheffield, J. M., \& Barch, D. M. (2016). Cognition and resting-state functional connectivity in schizophrenia. Neuroscience \& Biobehavioral Reviews, 61, 108-120.

Shinn, A. K., Baker, J. T., Lewandowski, K. E., Öngür, D., \& Cohen, B. M. (2015). Aberrant cerebellar connectivity in motor and association networks in schizophrenia. Front Hum Neurosci, 9.

Smesny, S., Gussew, A., Biesel, N. J., Schack, S., Walther, M., Rzanny, R., . . Schultz, C. C. (2015). Glutamatergic dysfunction linked to energy and membrane lipid metabolism in frontal and anterior cingulate cortices of never treated first-episode schizophrenia patients. Schizophr Res, 168(1), 322-329.

Smith, S. M., Fox, P. T., Miller, K. L., Glahn, D. C., Fox, P. M., Mackay, C. E., . . Laird, A. R. (2009). Correspondence of the brain's functional architecture during activation and rest. Proceedings of the National Academy of Sciences, 106(31), 13040-13045.

Spencer, K. M. (2011). Baseline gamma power during auditory steady-state stimulation in schizophrenia. Front Hum Neurosci, 5, 190. doi: 10.3389/fnhum.2011.00190

Stanghellini, G., Ballerini, M., Blasi, S., Mancini, M., Presenza, S., Raballo, A., \& Cutting, J. (2014). The bodily self: a qualitative study of abnormal bodily phenomena in persons with schizophrenia. Comprehensive psychiatry, 55(7), 1703-1711.

Stanghellini, G., Ballerini, M., Presenza, S., Mancini, M., Raballo, A., Blasi, S., \& Cutting, J. (2016). Psychopathology of Lived Time: Abnormal Time Experience in Persons With Schizophrenia. Schizophr Bull, 42(1), 45-55.

Stephan, K. E., Friston, K. J., \& Frith, C. D. (2009). Dysconnection in schizophrenia: from abnormal synaptic plasticity to failures of self-monitoring. Schizophr Bull, 35(3), 509-527.

Stevenson, R. A., Siemann, J. K., Schneider, B. C., Eberly, H. E., Woynaroski, T. G., Camarata, S. M., \& Wallace, M. T. (2014). Multisensory temporal integration in autism spectrum disorders. The Journal of Neuroscience, 34(3), 691-697. 
Su, T. W., Hsu, T. W., Lin, Y. C., \& Lin, C. P. (2015). Schizophrenia symptoms and brain network efficiency: A resting-state fMRI study. Psychiatry Res, 234(2), 208-218. doi: 10.1016/j.pscychresns.2015.09.013

Suazo, V., Lubeiro, A., Jurado-Barba, R., Moreno-Ortega, M., Dompablo, M., Morales-Muñoz, I., . . Molina, V. (2016). Elevated midline-parietal gamma band noise power in schizophrenia but not in bipolar patients. Eur Arch Psychiatry Clin Neurosci, 1-11.

Sun, L., Castellanos, N., Grutzner, C., Koethe, D., Rivolta, D., Wibral, M., . . . Uhlhaas, P. J. (2013). Evidence for dysregulated high-frequency oscillations during sensory processing in medication-naive, first episode schizophrenia. Schizophr Res, 150(2-3), 519-525. doi: 10.1016/j.schres.2013.08.023

Sun, M., Holter, S. M., Stepan, J., Garrett, L., Genius, J., Kremmer, E., . . Graw, J. (2013). Crybb2 coding for betaB2-crystallin affects sensorimotor gating and hippocampal function. Mamm Genome, 24(9-10), 333-348. doi: 10.1007/s00335-013-9478-7

Tan, H. R., Lana, L., \& Uhlhaas, P. J. (2013). High-frequency neural oscillations and visual processing deficits in schizophrenia. Front Psychol, 4, 621. doi: 10.3389/fpsyg.2013.00621

Taylor, S. F., Welsh, R. C., Chen, A. C., Velander, A. J., \& Liberzon, I. (2007). Medial frontal hyperactivity in reality distortion. Biol.Psychiatry, 61(10), 1171-1178.

Tu, P.-C., Lee, Y.-C., Chen, Y.-S., Li, C.-T., \& Su, T.-P. (2013). Schizophrenia and the brain's control network: aberrant within-and between-network connectivity of the frontoparietal network in schizophrenia. Schizophr Res, 147(2), 339-347.

Turgeon, M., Giersch, A., Delevoye-Turrell, Y., \& Wing, A. M. (2012). Impaired predictive timing with spared time interval production in individual with schizophrenia. Psychiatry research, 197(1), 13-18.

Uhlhaas, P. J., Linden, D. E., Singer, W., Haenschel, C., Lindner, M., Maurer, K., \& Rodriguez, E. (2006). Dysfunctional long-range coordination of neural activity during Gestalt perception in schizophrenia. J Neurosci, 26(31), 8168-8175. doi: 10.1523/JNEUROSCI.2002-06.2006

Uhlhaas, P. J., \& Singer, W. (2010). Abnormal neural oscillations and synchrony in schizophrenia. Nat Rev Neurosci, 11(2), 100-113. doi: 10.1038/nrn2774

Uhlhaas, P. J., \& Singer, W. (2012). Neuronal dynamics and neuropsychiatric disorders: toward a translational paradigm for dysfunctional large-scale networks. Neuron, 75(6), 963-980.

van Buuren, M., Vink, M., \& Kahn, R. S. (2012). Default-mode network dysfunction and selfreferential processing in healthy siblings of schizophrenia patients. Schizophr Res, 142(1), 237-243.

van der Meer, L., Costafreda, S., Aleman, A., \& David, A. S. (2010). Self-reflection and the brain: a theoretical review and meta-analysis of neuroimaging studies with implications for schizophrenia. Neurosci.Biobehav.Rev., 34(6), 935-946.

van der Meer, L., de Vos, A. E., Stiekema, A. P., Pijnenborg, G. H., van Tol, M. J., Nolen, W. A., . . . Aleman, A. (2013). Insight in schizophrenia: involvement of self-reflection networks? Schizophr Bull, 39(6), 1288-1295. doi: 10.1093/schbul/sbs122

van Wassenhove, V. (2009). Minding time in an amodal representational space. Philosophical Transactions of the Royal Society B: Biological Sciences, 364(1525), 1815-1830.

Vanhaudenhuyse, A., Demertzi, A., Schabus, M., Noirhomme, Q., Bredart, S., Boly, M., . . Laureys, S. (2011). Two distinct neuronal networks mediate the awareness of environment and of self. J Cogn Neurosci, 23(3), 570-578. doi: 10.1162/jocn.2010.21488

Wagner, G., De la Cruz, F., Schachtzabel, C., Güllmar, D., Schultz, C. C., Schlösser, R. G., . . . Koch, K. (2015). Structural and functional dysconnectivity of the fronto-thalamic system in schizophrenia: A DCM-DTI study. Cortex, 66, 35-45.

Walther, S. (2015). Psychomotor symptoms of schizophrenia map on the cerebral motor circuit. Psychiatry Research: Neuroimaging, 233(3), 293-298. 
Walther, S., \& Strik, W. (2012). Motor symptoms and schizophrenia. Neuropsychobiology, 66(2), 77-92.

Wang, X.-J., \& Krystal, J. H. (2014). Computational psychiatry. Neuron, 84(3), 638-654.

Weinberger, D. R., \& Radulescu, E. (2015). Finding the elusive psychiatric "lesion" with 21stcentury neuroanatomy: a note of caution. American Journal of Psychiatry, 173(1), 27-33.

White, R., \& Siegel, S. (2015). Cellular and circuit models of increased resting-state network gamma activity in schizophrenia. Neuroscience.

White, T. P., Joseph, V., O'Regan, E., Head, K. E., Francis, S. T., \& Liddle, P. F. (2010). Alphagamma interactions are disturbed in schizophrenia: a fusion of electroencephalography and functional magnetic resonance imaging. Clin Neurophysiol, 121(9), 1427-1437. doi: 10.1016/j.clinph.2010.03.024

Whitfield-Gabrieli, S., \& Ford, J. M. (2012). Default mode network activity and connectivity in psychopathology. Annu Rev Clin Psychol, 8, 49-76. doi: 10.1146/annurev-clinpsy-032511143049

Whitfield-Gabrieli, S., Moran, J. M., Nieto-Castañón, A., Triantafyllou, C., Saxe, R., \& Gabrieli, J. D. (2011). Associations and dissociations between default and self-reference networks in the human brain. Neuroimage, 55(1), 225-232.

Whitfield-Gabrieli, S., Thermenos, H. W., Milanovic, S., Tsuang, M. T., Faraone, S. V., McCarley, R. W., . . LaViolette, P. (2009). Hyperactivity and hyperconnectivity of the default network in schizophrenia and in first-degree relatives of persons with schizophrenia. Proceedings of the National Academy of Sciences, 106(4), 1279-1284.

Winterer, G., Ziller, M., Dorn, H., Frick, K., Mulert, C., Wuebben, Y., . . Coppola, R. (2000). Schizophrenia: reduced signal-to-noise ratio and impaired phase-locking during information processing. Clinical Neurophysiology, 111(5), 837-849.

Woodward, N. D., Karbasforoushan, H., \& Heckers, S. (2012). Thalamocortical dysconnectivity in schizophrenia. American Journal of Psychiatry.

Wotruba, D., Michels, L., Buechler, R., Metzler, S., Theodoridou, A., Gerstenberg, M., . . Heekeren, K. (2013). Aberrant coupling within and across the default mode, task-positive, and salience network in subjects at risk for psychosis. Schizophr Bull, sbt161.

Yang, G. J., Murray, J. D., Repovs, G., Cole, M. W., Savic, A., Glasser, M. F., . . Pearlson, G. D. (2014). Altered global brain signal in schizophrenia. Proceedings of the National Academy of Sciences, 111(20), 7438-7443.

Yang, G. J., Murray, J. D., Wang, X.-J., Glahn, D. C., Pearlson, G. D., Repovs, G., . . Anticevic, A. (2016). Functional hierarchy underlies preferential connectivity disturbances in schizophrenia. Proceedings of the National Academy of Sciences, 113(2), E219-E228.

Yu, Y., Shen, H., Zeng, L. L., Ma, Q., \& Hu, D. (2013). Convergent and divergent functional connectivity patterns in schizophrenia and depression. PLoS One, 8(7), e68250. doi: 10.1371/journal.pone.0068250

Zampini, M., Guest, S., Shore, D. I., \& Spence, C. (2005). Audio-visual simultaneity judgments. Perception \& psychophysics, 67(3), 531-544. 


\section{Psychopathological symptoms}

Abnormal spatiotemporal organisation of cognitive, affective, sensorimotor and social functions with subsequent behavioural abnormalities.

\section{Spatial features}

Abnormal connectivity and balance of relative activity levels between brain functional networks.

\section{Temporal features}

Abnormal oscillations and fluctuations at different frequencies and relationships between frequencies.

Figure 1: Alterations in the brain's spontaneous activity (lower panel) manifest themselves in altered spatial and temporal features (middle panel). These may lead to an altered construction/experience of time and space, in turn leading to psychopathological symptoms (upper panel) 


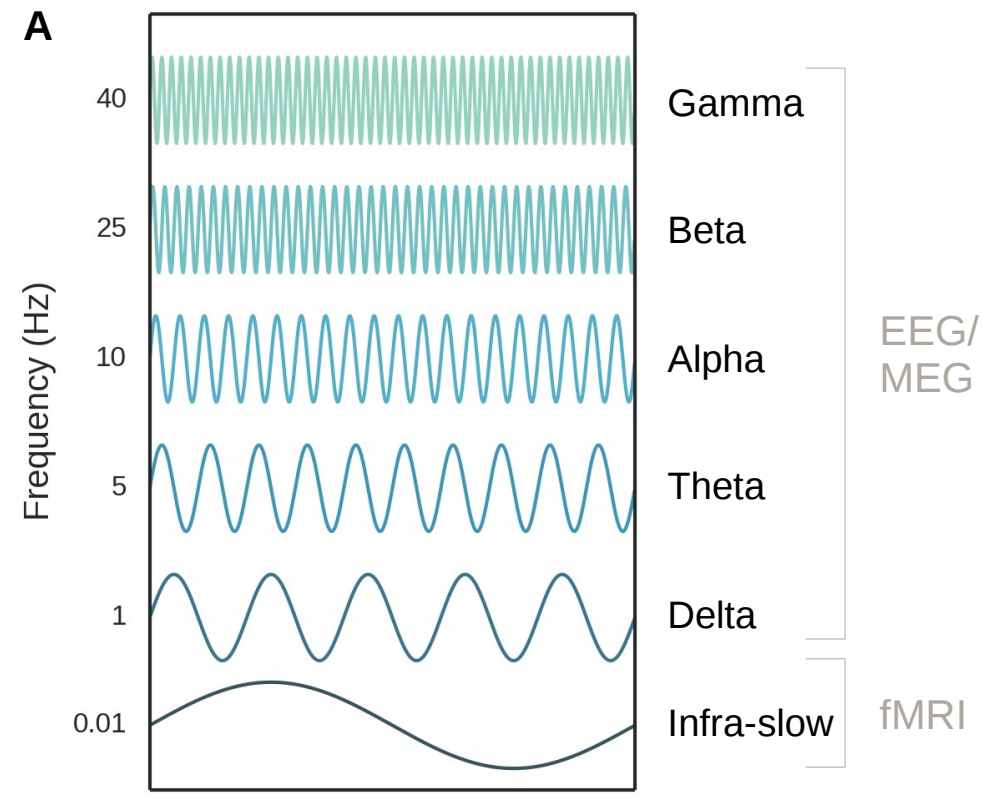

B

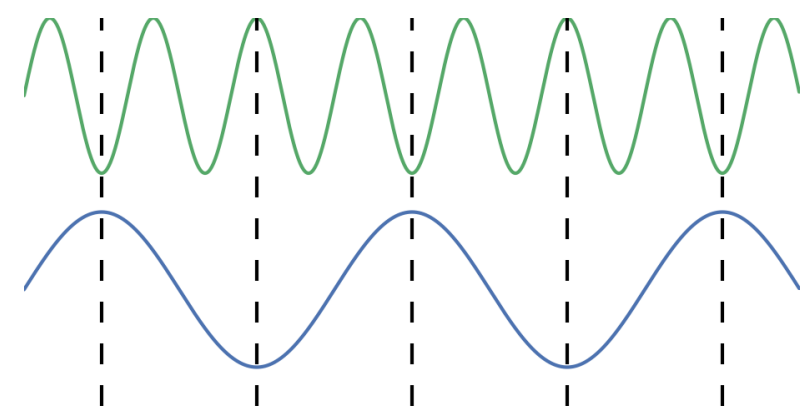

Figure 2: A description of what is meant by the different frequency bands and by cross-frequency coupling 
A

Healthy

Modal (and domain-dependent) perception and cognition of time within specific time windows

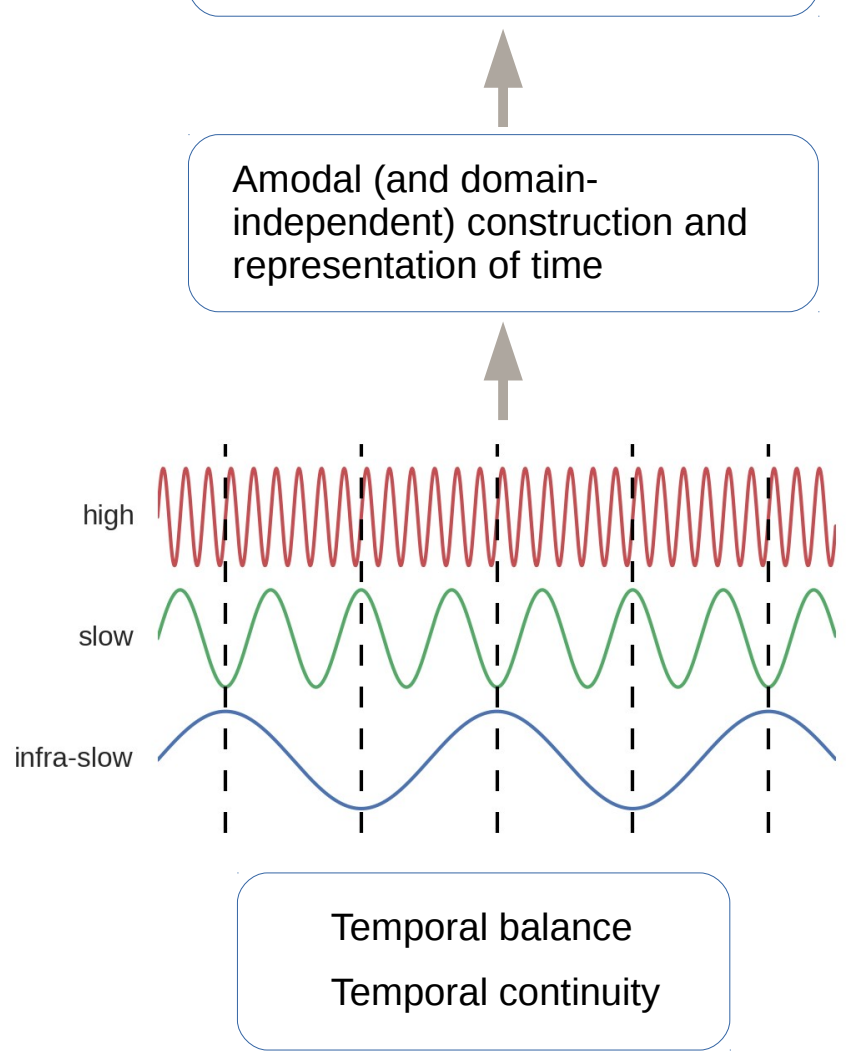

B

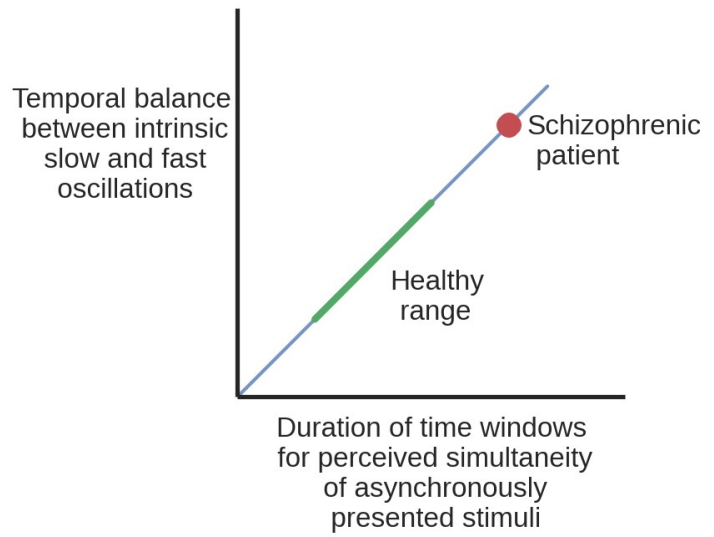

Schizophrenia

Enlarged time windows in perceived simultaneity and temporal order
Abnormal amodal (and domainindependent) construction and representation of time

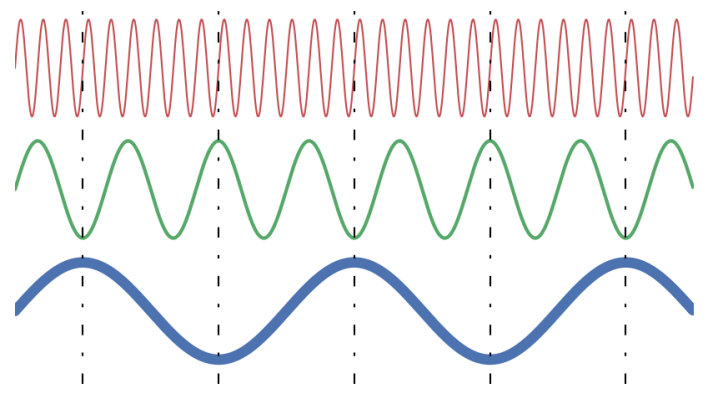

Temporal disbalance

Temporal fragmentation

C

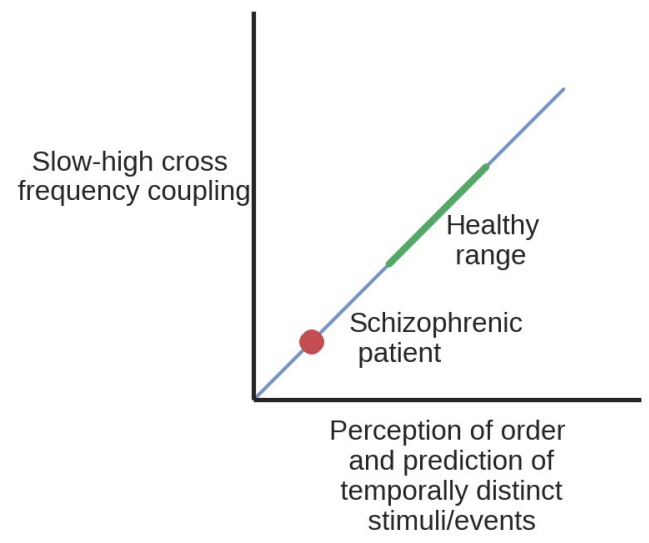

Figure 3: 


\section{Cognitive dysmetria}

Thought disturbances
Motor

Soft signs with small neurological signs

\section{Sensory}

Basic perceptual

symptoms
Cognitive-

attentional

deficits

Basic perceptual symptoms
Psychopathological symptoms

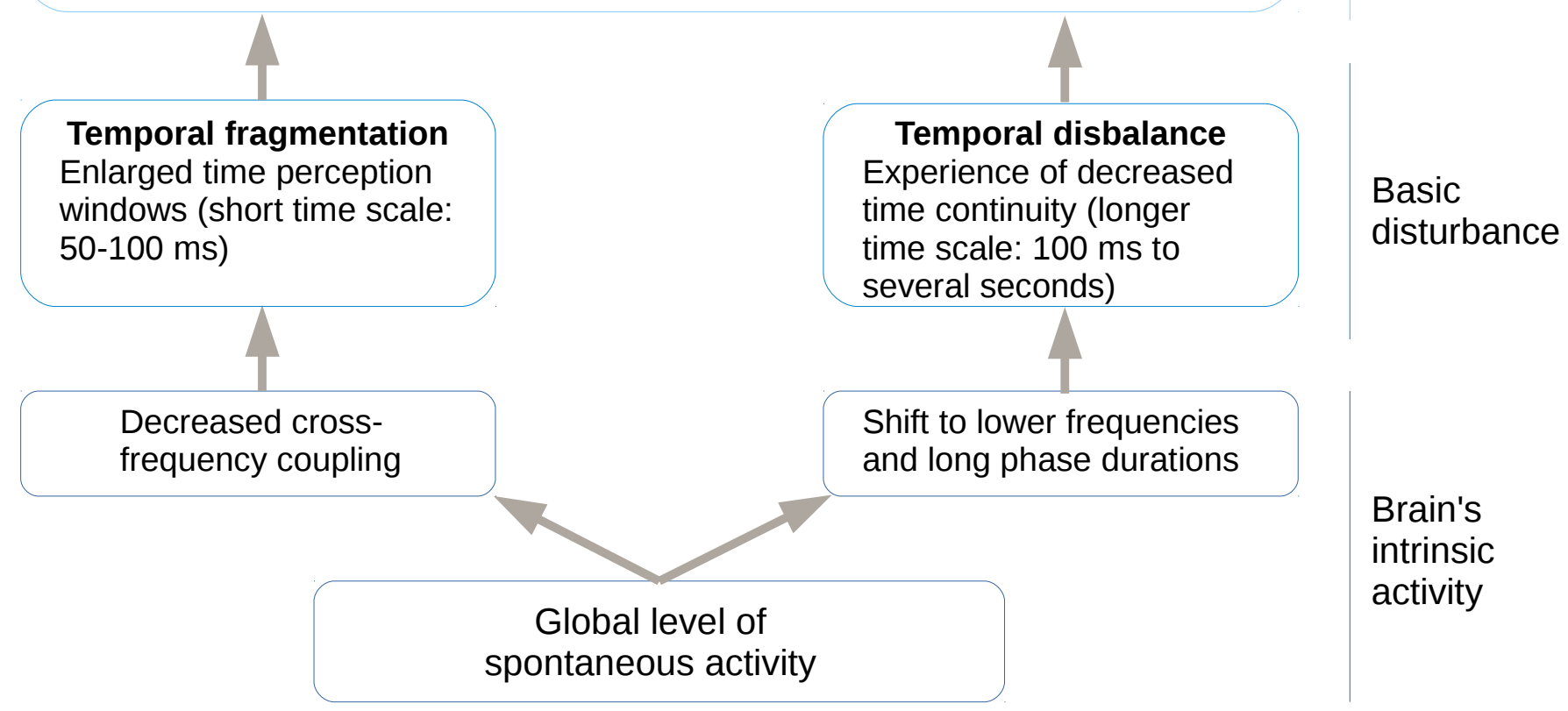

Figure 4: 
A
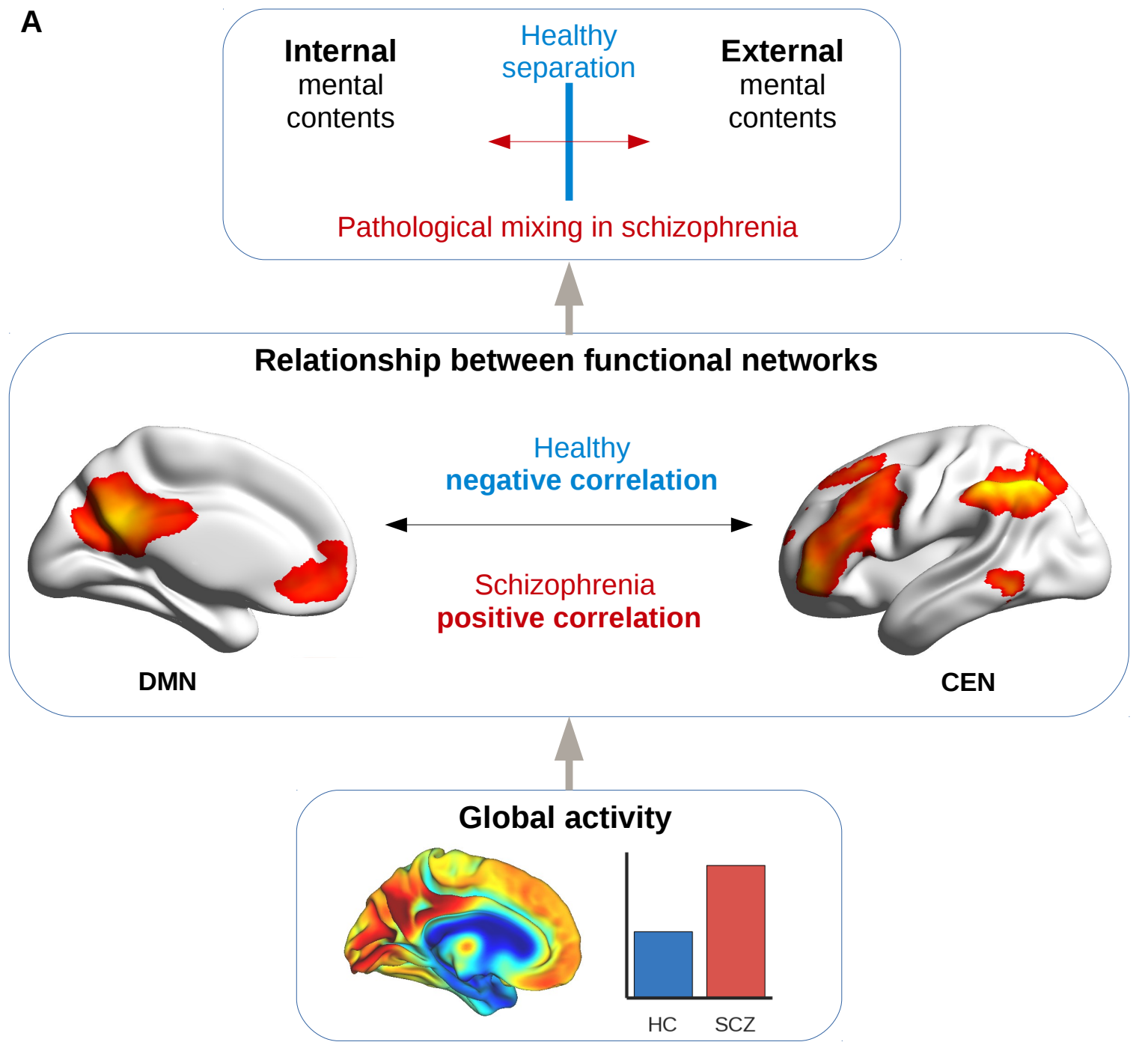

B

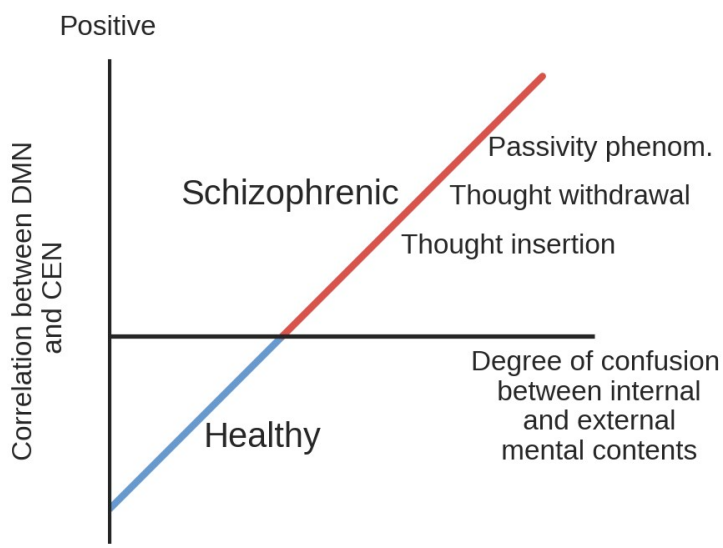

Negative 
Thought insertion, blockade and withdrawal
Self and identity disturbances and passivity phenomena

\section{Abnormal \\ Abnormal goal}

objectification of own body orientation
Psychopathological symptoms

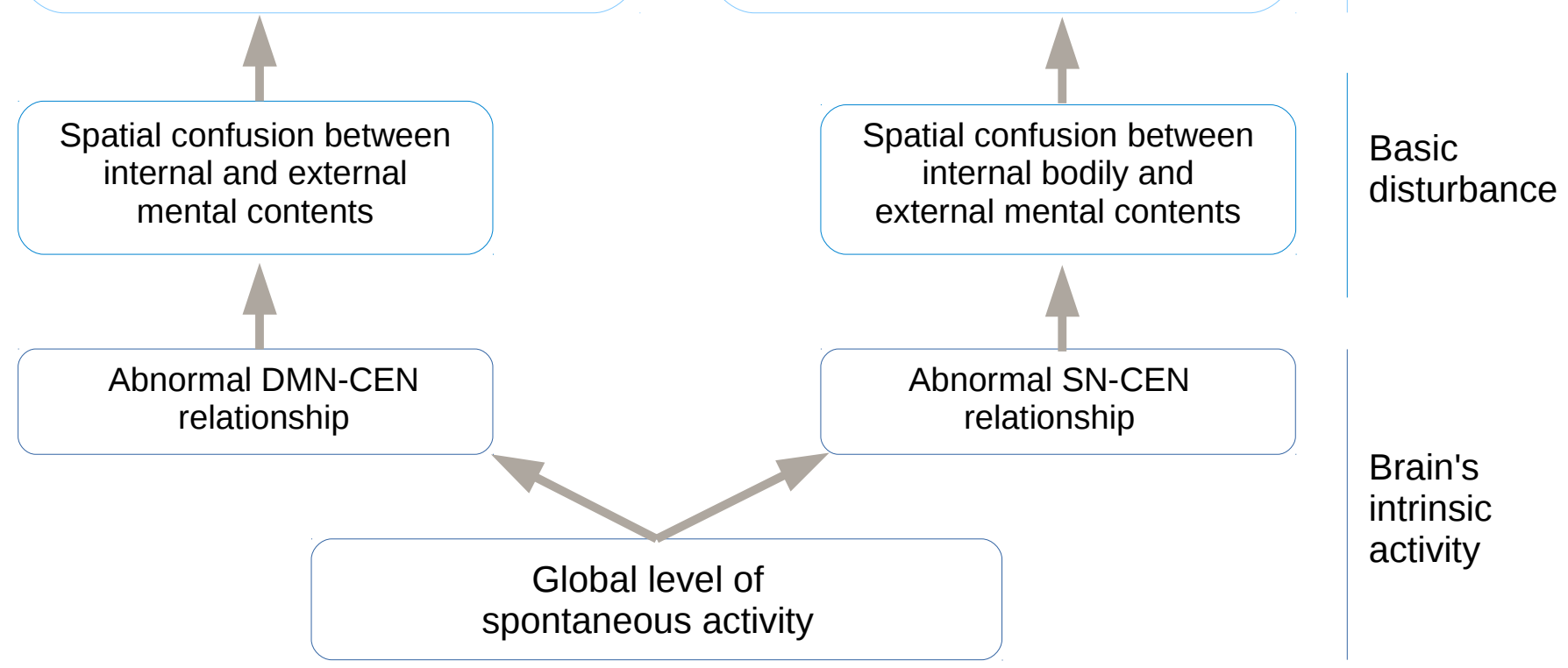


A

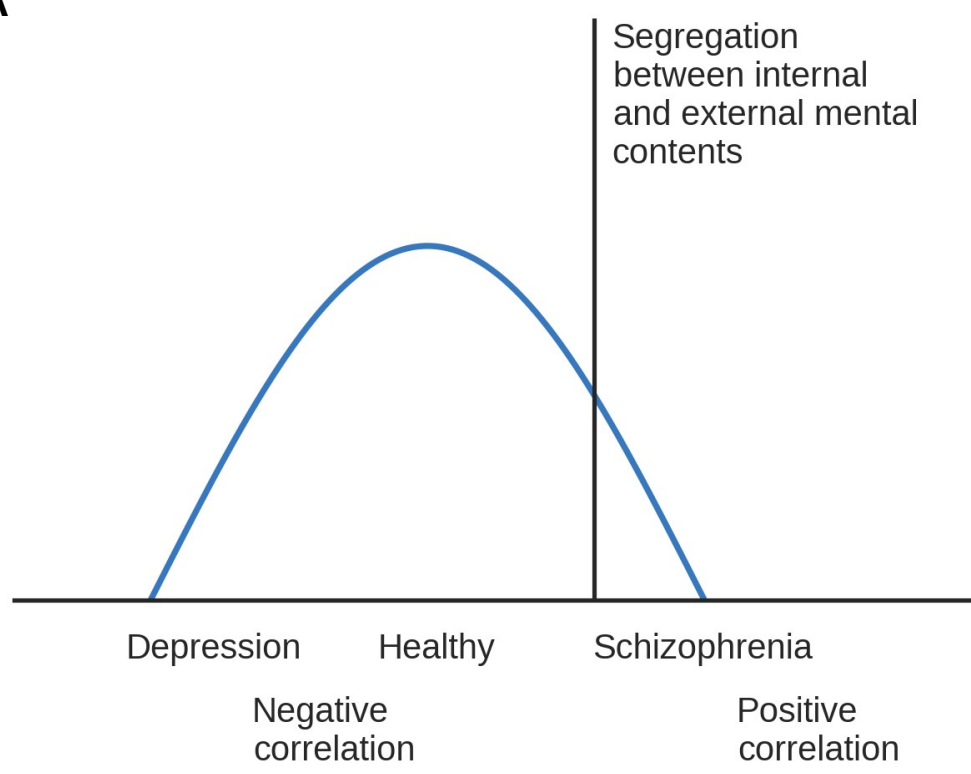

B

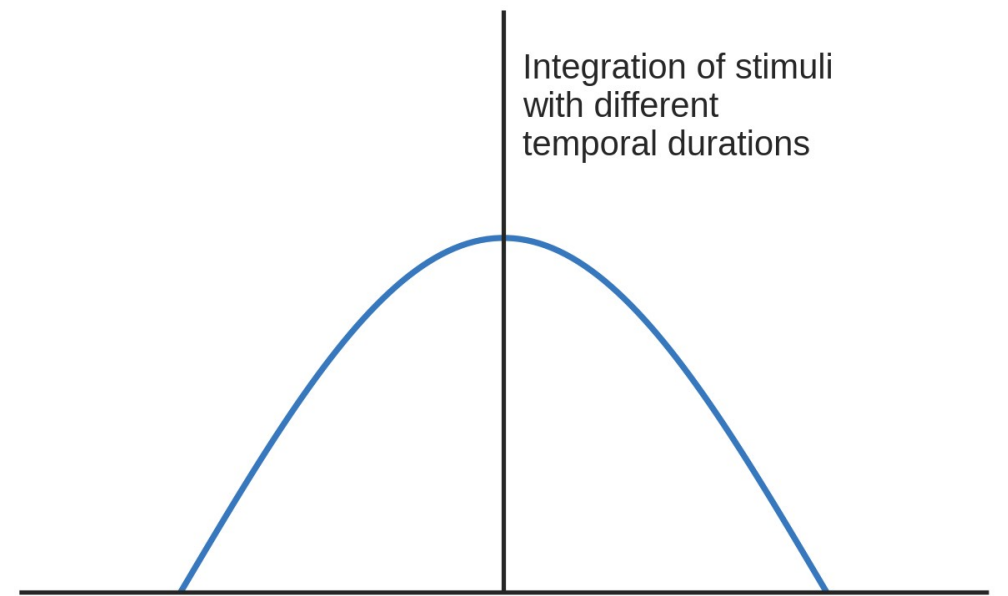

Schizophrenia

Healthy

Mania

Lower

frequencies
Correlation between

DMN and CEN

Balance in power between low and high frequencies (and associated cross frequency coupling) 


\section{Table 1 Key research challenges in resting state activity and psychiatry}

- Spatial structure: How do the different neural networks interact with each other? Are their balances and dysbalances related to particular psychiatric symptoms?

- Temporal structure: How are the oscillations and fluctuations in different frequency bands related to each other? Is there phase-power coupling from lower to higher frequencies and how are its disturbances surface in psychiatric symptoms?

- How can we develop spatial and temporal measures of psychiatric symptoms? Do we for instance have to conceive hallucinations in temporal and spatial terms rather than in terms of specific contents like linguistic as related to the voices?

- How can we develop valid and reliable method for apprehending the temporal and spatial features of psychiatric symptoms? Do we need to consider the patients' subjective experience of the temporal and spatial features of their psychiatric symptoms (see Northoff 2014, Woods 2015, Stanghellini et al. 2015)?

- Do the resting state's abnormal spatial and temporal structuring also apply to affective, cognitive, social and sensorimotor functions whose task-evoked activity is based on the resting state? If so do we need to understand the psychiatric patients' cognitive, affective, social and sensorimotor abnormalities as primarily spatial and temporal rather than cognitive, affective, sensorimotor, and social? How can we measure such spatiotemporal abnormalities in cognitive, affective, sensorimotor, and social functions?

- How does the resting state activity interact with specific stimuli or tasks? Is this interaction linear and therefore merely additive or rather non-linear and henceforth non-additive (Northoff et al. 2010, He 2013, Sadaghiani et al. 2010, Northoff 2014b)? How can we distinguish in psychiatric patients whether their resting state itself is abnormal or whether it is the rest-stimulus interaction that leads to the abnormal symptoms?

- How are early life experiences related to the constitution of the resting state's spatiotemporal structure in its neural activity? Can we trace neurodevelopmental 
disorders like schizophrenia back to the early life experiences' impact on the resting state?

- How is the resting state activity and its spatial and temporal features related to biochemical modulation by for instance GABA and Glutamate (see Duncan et al. 2014), the excitation-inhibition balance, and other transmitters? How do related and other genes control the individual expression and manifestation of the resting state activity and especially its spatiotemporal features? 